\title{
APLICAÇÃO DE AMIDOXIMAS COMO CATALISADORES DA REAÇÃO DE ALILAÇÃO POR ALILTRIFLUOROBORATO DE POTÁSSIO EM MEIO BIFÁSICO
}

\section{Dmistocles de Andrade ${ }^{a}$, João Rufino de Freitas Filho e Juliano Carlo Rufino Freitas ${ }^{\text {a,b,* }}$}

aDepartamento de Química, Universidade Federal de Rural de Pernambuco, Av. Dom Manoel de Medeiros, s/n, 52171-900, Recife - PE, Brasil

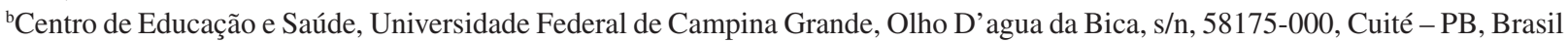

APPLICATION OF AMIDOXIMES AS CATALYSTS FOR THE ALLYLATION REACTION BY POTASSIUM ALLYLTRIFLUOROBORATE IN ORGANIC BIPHASIC. We describe the application of an amidoxime as catalyst in the synthesis of homoallylic alcohols from the allylation reaction of aldehydes with different features using potassium allyltrifluoroborate. The reaction was conducted with a catalytic amount of the appropriate amidoxime in a biphasic system (water:dichloromethane), open atmosphere and ambient temperature. The products were obtained in yields ranging from moderate to excellent in short reaction times without the need for further purifications for almost all examples. Additionally, the reaction has proved chemo- and regioselective.

Keywords: allylation; homoallylic alcohols; amidoximes; potassium allyltrifluoroborate

\section{INTRODUÇÃO}

As amidoximas são importantes e versáteis blocos de construção para a síntese de vários núcleos heterocíclicos como oxadiazol, ${ }^{1}$ triazol, ${ }^{2}$ quinazolinas, ${ }^{3}$ entre outros. ${ }^{4}$ Adicionalmente, estes compostos apresentam uma gama de aplicações biológicas/farmacológicas ${ }^{5}$ e tecnológicas, ${ }^{6}$ em parte devido às amidoximas atuarem como pró-fármacos das amidinas ${ }^{7}$ e por poderem complexar com diferentes íons metálicos. ${ }^{8}$ Recentemente, foi descrito a síntese e o uso de uma resina amidoxímica complexada com $\mathrm{Pd}(\mathrm{II})$ como agente promotor da reação de formação de novas ligações C-C em um acoplamento cruzado do tipo Suzuki. ${ }^{9}$

Dentre os inúmeros métodos para formação de novas ligações C-C, o mais amplamente utilizado é a reação de alilação e crotilação de compostos carbonílicos. ${ }^{10}$ Os produtos resultantes desta reação são os alcoóis homoalílicos, importantes precursores sintéticos na preparação de diversos produtos naturais e moléculas biologicamente ativas como, por exemplo, a $(S)$-Tiloforina, ${ }^{11}$ Rifamicina $S,{ }^{12}$ Epotilona $\mathrm{D},{ }^{13}$ (+)-Antimicina A, ${ }^{14}$ (R)-Goniotalamina ${ }^{15}$ e Hemibrovetoxina $\mathrm{B}^{16}$ (Figura 1).<smiles>COc1cc2c3c(c4cc(OC)c(OC)cc4c2cc1OC)CN1CCC[C@H]1C3</smiles>

(S)-Tiloforina

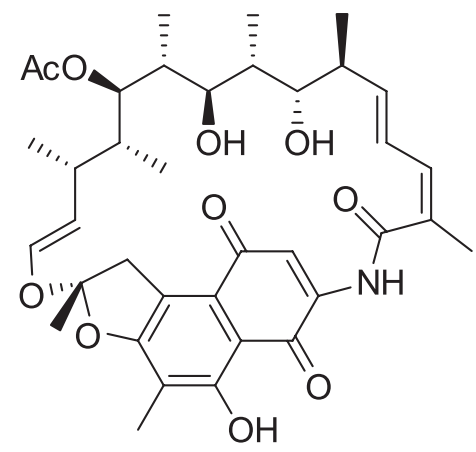

Rifamicina S<smiles>CCCCCCCCCCCCCCCCCC(=O)O[C@@H]1C(=O)O[C@H](C)[C@@H](NC(=O)c2cccc(NC=O)c2O)C(=O)O[C@@H]1C</smiles>

(+)-Antimicina A<smiles></smiles>

Epotilona D<smiles>O=C1C=CC[C@H](/C=C/c2ccccc2)O1</smiles>

(R)-Goniotalamina

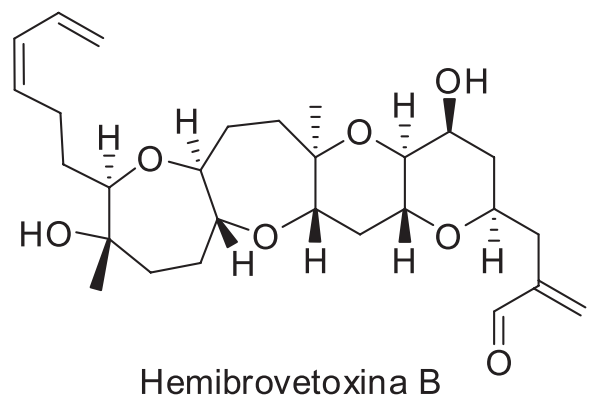

Figura 1. Exemplos de produtos naturais obtidos a partir dos alcoóis homoalílicos 
Na literatura, é comum encontrar relatos da reação de alilação de compostos carbonílicos empregando-se espécies organometálicas alílicas de $\mathrm{Li}$ ou $\mathrm{Mg},{ }^{17}$ os quais apresentam uma elevada nucleofilicidade (comprometendo a quimiosseletividade da reação quando o composto carbonílico apresenta outros grupos funcionais) e são instáveis ao ar e água (o que limita seu uso em condições verdes). Uma alternativa a estas desvantagens é o uso dos sais de trifluoroboratos orgânicos neste tipo de reação. Estes reagentes são estáveis ao ar e termoestáveis, de fácil manipulação e podem ser estocados por longos períodos sem apresentar degradação. Além disso, por serem sais, a sua utilização possibilita o emprego da água como um dos solventes da reação. ${ }^{18}$ Adicionalmente, os subprodutos desta reação são compostos inorgânicos de boro, atóxicos e solúveis em água. ${ }^{18}$

Embora a literatura cite diversas metodologias para a alilação de compostos carbonílicos empregando sais orgânicos de trifluoroboratos utilizando ou não promotores ou catalisadores, ${ }^{19}$ esta reação apresenta alguns desafios relacionados ao emprego da água como cossolvente e controle da estereosseletividade, entre outros fatores.

Diante do exposto, este trabalho descreve a síntese de diferentes álcoois homoalílicos através da reação de alilação de aldeídos por organotrifluoroborato de potássio mediada por aril-amidoximas utilizando um sistema bifásico.

\section{PARTE EXPERIMENTAL}

\section{Generalidades}

Os solventes foram purificados de acordo com os métodos descritos na literatura. ${ }^{20} \mathrm{O}$ hexano e o acetato de etila foram destilados com coluna de vigreaux, o diclorometano foi destilado sob $\mathrm{CaH}_{2}$, o etanol foi destilado sob magnésio metálico. Os solventes foram evaporados em um evaporador rotativo da Büchi Rotavapor modelo R-114 conectado a uma bomba de vácuo modelo KNF Neuberger, e o solvente remanescente foi eliminado utilizando uma bomba de alto vácuo da Edwards modelo RV3.

Os espectros de ressonância magnética nuclear foram registrados em um espectrômetro Varian Unity Plus de $300 \mathrm{MHz}$ ou em um espectrômetro Varian URMNS de 400 MHz. Os deslocamentos químicos estão expressos em ppm (partes por milhão) em relação ao pico residual do clorofórmio (7,25 ppm) ou dimetilsulfóxido (2,50 ppm) para os espectros de hidrogênio, em relação ao pico central do $\mathrm{CDCl}_{3}$ (77,0 ppm) ou DMSO- $d_{6}(39,7 \mathrm{ppm})$ para os espectros de carbono. Todas as constantes de acoplamento $(J)$ foram descritas em hertz $(\mathrm{Hz})$.

Os espectros de infravermelho foram registrados em um em espectrofotômetro de IV com transformada de Fourier no instrumento Bruker Modelo IFS66, sendo as amostras preparadas como filmes finos ou pastilhas de $\mathrm{KBr}$.

Os pontos de fusão foram realizados no Electro-thermal série IA 9100 Digital Melting Point.

As purificações por cromatografia em coluna foram efetuadas utilizando-se sílica-gel (230-400 mesh) seguindo o método descrito por Still e colaboradores. ${ }^{21}$ A cromatografia em camada delgada (CCD) foi realizada utilizando placas de sílica-gel contendo indicador fluorescente F254 da Merck. Para visualização, as placas foram colocadas em luz ultravioleta, vapor de iodo, solução ácida (EtOH/ $\left.\mathrm{H}_{2} \mathrm{SO}_{4}, 95: 5\right)$ e solução de vanilina.

\section{Procedimento para preparação das aril-amidoximas $\mathbf{2 a - h ^ { 2 2 }}$}

Em um balão de fundo redondo de $50 \mathrm{~mL}$ contendo a aril-nitrila apropriada 1a-h $(2,5 \mathrm{mmol})$ dissolvida em etanol $(5 \mathrm{~mL})$, foi adicionada uma solução aquosa contendo cloridrato de hidroxilamina (518 mg; 7,5 mmol) e carbonato de sódio (392 mg; 3,7 mmol) dissolvido em $5 \mathrm{~mL}$ de água. Esta mistura reacional foi irradiada por ultrassom, a uma temperatura de $50{ }^{\circ} \mathrm{C}$, pelo tempo descrito na Tabela 1 . O término da reação foi verificado através de cromatografia de camada delgada (CCD) e a mistura reacional foi concentrada por evaporação rotativa sob pressão reduzida. Ao sistema bifásico formado foi adicionado acetato de etila $(50 \mathrm{~mL})$ e a solução foi então lavada com solução saturada de cloreto de amônio $(2 \times 25 \mathrm{~mL})$. A fase orgânica foi separada, seca sob $\mathrm{MgSO}_{4}$ anidro e filtrada. $\mathrm{O}$ solvente foi removido por evaporação rotativa à pressão reduzida e cristalizado utilizando clorofórmio/hexano (90:10) como solventes para levar às aril-amidoximas (2a-h) desejadas em rendimentos que variaram de 35 a $91 \%$.

Benzamidoxima (2a): Obtido 0,27 g (82\%); sólido branco, P.F. 79-80 ${ }^{\circ} \mathrm{C}$ (lit. ${ }^{22}$ P.F. $78,5^{\circ} \mathrm{C}$ ); IV (Pastilha de $\mathrm{KBr}$ ): v 3453, 361, 3057 , 2369, 2293, 1649, 1529, 1387, 927, $691 \mathrm{~cm}^{-1}$. RMN ${ }^{1} \mathrm{H}$ (DMSO- $d_{6}$, $400 \mathrm{MHz}): \delta 9,63$ (s, 1H, OH); 7,69-7,67 (m, 2H, $\left.\mathrm{H}_{\text {aromático }}\right) ; 7,38-$ 7,35 (m, 3H, $\left.\mathrm{H}_{\text {aromático }}\right) ; 5,80$ (s, 2H, $\mathrm{NH}_{2}$ ). RMN ${ }^{13} \mathrm{C}$ (DMSO- $d_{6}, 100$ $\mathrm{MHz}): \delta 151,0 ; 133,5 ; 129,0 ; 128,2 ; 125,5$.

2-Metilbenzamidoxima (2b): Obtido 0,13 g (35\%); sólido branco, P.F. $145-147{ }^{\circ} \mathrm{C}$ (lit. ${ }^{22}$ P.F. $145-146{ }^{\circ} \mathrm{C}$ ); IV (Pastilha de $\mathrm{KBr}):$ v 3479, 3362, 2918, 2356, 1655, 1588, 1377, 905, $771 \mathrm{~cm}^{-1}$. RMN ${ }^{1} \mathrm{H}$ (DMSO- $\left.d_{6}, 400 \mathrm{MHz}\right): \delta 9,30(\mathrm{~s}, 1 \mathrm{H}, \mathrm{OH}) ; 7,29-7,26(\mathrm{~m}$, $\left.2 \mathrm{H}, \mathrm{H}_{\text {aromático }}\right) ; 7,22-7,16\left(\mathrm{~m}, 2 \mathrm{H}, \mathrm{H}_{\text {aromático }}\right) ; 5,71\left(\mathrm{~s}, 2 \mathrm{H}, \mathrm{NH}_{2}\right) ; 2,35$ (s, $\left.3 \mathrm{H}, \mathrm{CH}_{3}\right) . \mathrm{RMN}{ }^{13} \mathrm{C}$ (DMSO- $\left.d_{6}, 100 \mathrm{MHz}\right): \delta 152,4 ; 136,4 ; 134,4$; 130,$2 ; 129,0 ; 128,6 ; 125,5 ; 19,9$.

3-Metilbenzamidoxima (2c): Obtido 0,30 g (81\%); sólido branco, P.F. 91-92 ${ }^{\circ} \mathrm{C}$ (lit. ${ }^{22}$ P.F. 91-91,5 ${ }^{\circ} \mathrm{C}$ ); IV (Pastilha de $\mathrm{KBr}$ ): $v$ 3452, 3349, 3042, 2370, 2278, 1648, 1588, 1386, 933, 894, 707 $\mathrm{cm}^{-1}$. RMN ${ }^{1} \mathrm{H}$ (DMSO- $\left.d_{6}, 400 \mathrm{MHz}\right): \delta 9,57$ (s, 1H, OH); 7,50 (sl, $\left.1 \mathrm{H}, \mathrm{H}_{\text {aromático }}\right) ; 7,46\left(\mathrm{~d}, 1 \mathrm{H}, J=8,0 \mathrm{~Hz}, \mathrm{H}_{\text {aromático }}\right) ; 7,25$ (dd, 1H, $J=$ 8,0 e 7,2 Hz, $\left.\mathrm{H}_{\text {aromático }}\right) ; 7,17\left(\mathrm{~d}, 1 \mathrm{H}, J=7,2 \mathrm{~Hz}, \mathrm{H}_{\text {aromático }}\right) ; 5,75(\mathrm{~s}, 2 \mathrm{H}$, $\mathrm{NH}_{2}$ ); 2,31 (s, $\left.3 \mathrm{H}, \mathrm{CH}_{3}\right)$. RMN ${ }^{13} \mathrm{C}$ (DMSO- $\left.d_{6}, 100 \mathrm{MHz}\right): \delta 151,1$; 137,$3 ; 133,5 ; 129,7 ; 128,1 ; 126,1 ; 122,7 ; 21,2$.

4-Metilbenzamidoxima (2d): Obtido 0,33 g (88\%); sólido branco, P.F. $145-146{ }^{\circ} \mathrm{C}$ (lit. ${ }^{22}$ P.F. $146{ }^{\circ} \mathrm{C}$ ); IV (Pastilha de $\mathrm{KBr}$ ): v $3499,3370,3054,1918,1667,1588,1391,937,825,751 \mathrm{~cm}^{-1}$. RMN ${ }^{1} \mathrm{H}$ (DMSO- $\left.d_{6}, 400 \mathrm{MHz}\right): \delta 9,52(\mathrm{~s}, 1 \mathrm{H}, \mathrm{OH}) ; 7,56$ (d, 2H, $J=8,4$ $\left.\mathrm{Hz}, \mathrm{H}_{\text {aromático }}\right) ; 7,16\left(\mathrm{~d}, 2 \mathrm{H}, J=8,4 \mathrm{~Hz}, \mathrm{H}_{\text {aromático }}\right) ; 5,73\left(\mathrm{~s}, 2 \mathrm{H}, \mathrm{NH}_{2}\right.$ ); $2,30\left(\mathrm{~s}, 3 \mathrm{H}, \mathrm{CH}_{3}\right) . \mathrm{RMN}^{13} \mathrm{C}\left(\mathrm{DMSO}-d_{6}, 100 \mathrm{MHz}\right): \delta 150,5 ; 138,0$; 130,$3 ; 128,4 ; 125,0 ; 20,5$.

4-Bromobenzamidoxima (2e): Obtido 0,45 g (85\%); sólido branco, P.F. $136-137^{\circ} \mathrm{C}$ (lit. ${ }^{22}$ P.F. $135-136{ }^{\circ} \mathrm{C}$ ); IV (Pastilha de $\mathrm{KBr}$ ); $v 3474,3356,3150,2361,1657,1587,1388,919,835,740 \mathrm{~cm}^{-1}$. RMN ${ }^{1} \mathrm{H}$ (DMSO- $\left.d_{6}, 400 \mathrm{MHz}\right): \delta 9,73(\mathrm{~s}, 1 \mathrm{H}, \mathrm{OH}) ; 7,62(\mathrm{~d}, 2 \mathrm{H}, J=8,8$ $\left.\mathrm{Hz}, \mathrm{H}_{\text {aromático }}\right) ; 7,56$ (d, 2H, $\left.J=8,8 \mathrm{~Hz}, \mathrm{H}_{\text {aromático }}\right) ; 5,85$ (s, 2H, $\mathrm{NH}_{2}$ ). RMN ${ }^{13} \mathrm{C}\left(\mathrm{DMSO}-d_{6}, 100 \mathrm{MHz}\right): \delta 150,1 ; 132,7 ; 131,2 ; 127,6 ; 122,2$.

4-Clorobenzamidoxima (2f): Obtido $0,34 \mathrm{~g}(80 \%)$; sólido branco, P.F. $133-135^{\circ} \mathrm{C}$ (lit. ${ }^{22}$ P.F. $133{ }^{\circ} \mathrm{C}$ ); IV (Pastilha de KBr): v 3478, $3372,3153,1659,1492,1414,1097,1016,965,802,736 \mathrm{~cm}^{-1}$. RMN ${ }^{1} \mathrm{H}$ (DMSO-d $\left.d_{6}, 400 \mathrm{MHz}\right): \delta 9,73(\mathrm{~s}, 1 \mathrm{H}, \mathrm{OH}) ; 7,69(\mathrm{~d}, 2 \mathrm{H}, J=8,4$ $\left.\mathrm{Hz}, \mathrm{H}_{\text {aromático }}\right) ; 7,43\left(\mathrm{~d}, 2 \mathrm{H}, J=8,4 \mathrm{~Hz}, \mathrm{H}_{\text {aromático }}\right) ; 5,82\left(\mathrm{~s}, 2 \mathrm{H}, \mathrm{NH}_{2}\right)$. RMN ${ }^{13} \mathrm{C}$ (DMSO- $\left.d_{6}, 100 \mathrm{MHz}\right): \delta 149,7 ; 133,2 ; 131,9 ; 127,9 ; 126,9$.

4-Piridinoamidoxima (2g): Obtido 0,28 g (81\%); sólido branco, P.F. $194-196{ }^{\circ} \mathrm{C}$ (lit. ${ }^{22}$ P.F. $209{ }^{\circ} \mathrm{C}$ ); IV (Pastilha de KBr): v 3459, $3312,3101,2464,2364,1633,1599,1420,1387,947,664 \mathrm{~cm}^{-1}$. RMN ${ }^{1} \mathrm{H}$ (DMSO- $\left.d_{6}, 400 \mathrm{MHz}\right): \delta 10,11$ (s, 1H, OH); 8,57 (d, 2H, $J=6,4$ 
$\left.\mathrm{Hz}, \mathrm{H}_{\text {heteroaromático }}\right) ; 7,64$ (d, 2H, $\left.J=6,4 \mathrm{~Hz}, \mathrm{H}_{\text {heteroaromático }}\right) ; 6,01$ (s, 2H, $\mathrm{NH}_{2}$ ). RMN ${ }^{13} \mathrm{C}$ (DMSO- $\left.d_{6}, 100 \mathrm{MHz}\right): \delta 149,9 ; 149,1 ; 140,7 ; 119,8$.

4-Nitrobenzamidoxima (2g): Obtido 0,41 g (91\%); sólido amarelo, P.F. $168-170{ }^{\circ} \mathrm{C}$ (lit. ${ }^{22}$ P.F. $169,2-169,9{ }^{\circ} \mathrm{C}$ ); IV (Pastilha de $\mathrm{KBr}):$ v 3463, 3358, 3111, 1662, 1602, 1517, 1341, 927, 864, 704 $\mathrm{cm}^{-1} . \mathrm{RMN}{ }^{1} \mathrm{H}\left(\mathrm{DMSO}-d_{6}, 400 \mathrm{MHz}\right): \delta 10,13(\mathrm{~s}, 1 \mathrm{H}, \mathrm{OH}) ; 8,22(\mathrm{~d}$, $\left.2 \mathrm{H}, J=8,8 \mathrm{~Hz}, \mathrm{H}_{\text {aromático }}\right) ; 7,94\left(\mathrm{~d}, 2 \mathrm{H}, J=8,8 \mathrm{~Hz}, \mathrm{H}_{\text {aromático }}\right) ; 6,06$ (s, $\left.2 \mathrm{H}, \mathrm{NH}_{2}\right) . \mathrm{RMN}{ }^{13} \mathrm{C}$ (DMSO- $\left.d_{6}, 100 \mathrm{MHz}\right): \delta 149,6 ; 147,6 ; 139,7$; 126,$6 ; 123,5$.

Procedimento para alilação de aldeídos por aliltrifluoroborato de potássio (3) catalisada pela 4-bromobenzamidoxima (2e $)^{19}$

Em um balão de fundo redondo contendo o aldeído apropriado 4a-x $(0,5 \mathrm{mmol})$ em diclorometano $(2 \mathrm{~mL})$, foi adicionada a 4-bromobenzamidoxima (2e, $5,3 \mathrm{mg}$; 0,025 mmol; $5 \mathrm{~mol} \%$ ), o aliltrifluoroborato de potássio $(\mathbf{3}, 89 \mathrm{mg} ; 0,6 \mathrm{mmol})$ e a água $(2 \mathrm{~mL})$. A mistura foi deixada sob agitação e monitorada por CCD, utilizando como sistema de eluição uma mistura de hexano/AcOEt (7:3). Ao término da reação foi adicionado AcOEt $(15 \mathrm{~mL})$ e a mistura foi lavada com uma solução saturada de bicarbonato de potássio $(3 \times 15 \mathrm{~mL})$. A fase orgânica foi separada, seca sob $\mathrm{MgSO}_{4}$ anidro e filtrada. $\mathrm{O}$ solvente foi removido sob vácuo fornecendo os álcoois homoalílicos $\mathbf{5 a - x}$ sem a necessidade de purificação posterior.

1-(Naftalen-2-il)but-3-en-1-ol (5a): Obtido $90 \mathrm{mg}(91 \%)$; RMN ${ }^{1} \mathrm{H}\left(300 \mathrm{MHz}, \mathrm{CDCl}_{3}\right) \delta$ 7,86-7,80 (m, 4H, $\left.\mathrm{H}_{\text {aromático }}\right) ; 7,54-7,46$ (m, 3H, $\mathrm{H}_{\text {aromático }}$ ); 5,84 (ddt, $1 \mathrm{H}, J=17,1 ; 10,2$ e $\left.7,5 \mathrm{~Hz}, \mathrm{CH}=\mathrm{CH}_{2}\right) ; 5,25-5,14$ $\left(\mathrm{m}, 2 \mathrm{H}, \mathrm{CH}=\mathrm{C}_{2}\right) ; 4,91$ (dd, $1 \mathrm{H}, J=7,2$ e $\left.5,1 \mathrm{~Hz}, \mathrm{CHOH}\right) ; 2,63-2,56$ $\left(\mathrm{m}, 2 \mathrm{H}, \mathrm{CHCH}_{2}\right) ; 2,10(\mathrm{sl}, 1 \mathrm{H}, \mathrm{OH}) . \mathrm{RMN}^{13} \mathrm{C}\left(75 \mathrm{MHz}, \mathrm{CDCl}_{3}\right) \delta$ 141,$1 ; 134,2 ; 133,2 ; 132,8 ; 128,1 ; 127,8 ; 127,5 ; 126,0 ; 125,8 ; 124,2$; 123,$9 ; 118,4 ; 73,3 ; 43,6$.

1-Fenil-but-3-en-1-ol (5b): Obtido $60 \mathrm{mg}(81 \%)$; $\mathrm{RMN}{ }^{1} \mathrm{H}$ $\left(300 \mathrm{MHz}, \mathrm{CDCl}_{3}\right) \delta 7,36-7,23\left(\mathrm{~m}, 5 \mathrm{H}, \mathrm{H}_{\text {aromático }}\right) ; 5,87-5,72(\mathrm{~m}, 1 \mathrm{H}$, $\left.\mathrm{C} \underline{\mathrm{H}}=\mathrm{CH}_{2}\right) ; 5,18-5,11\left(\mathrm{~m}, 2 \mathrm{H}, \mathrm{CH}=\mathrm{CH}_{2}\right) ; 4,73(\mathrm{dd}, 1 \mathrm{H}, J=7,5$ e $5,4 \mathrm{~Hz}$, $\mathrm{C} \underline{\mathrm{HOH}}) ; 2,54-2,47$ (m, 2H, $\left.\mathrm{CHC}_{2}\right) ; 2,00(\mathrm{sl}, 1 \mathrm{H}, \mathrm{OH}) . \mathrm{RMN}^{13} \mathrm{C}(75$ $\left.\mathrm{MHz}, \mathrm{CDCl}_{3}\right) \delta 143,7 ; 134,3 ; 128,2 ; 127,3 ; 125,7 ; 118,0 ; 73,2 ; 43,5$.

1-4-tolylbut-3-en-1-ol (5c): Obtido $75 \mathrm{mg}(93 \%), \mathrm{RMN}{ }^{1} \mathrm{H}$ (300 MHz, $\left.\mathrm{CDCl}_{3}\right): \delta 7,23\left(\mathrm{~d}, 2 \mathrm{H}, J=7,8 \mathrm{~Hz}, \mathrm{H}_{\text {aromático }}\right) ; 7,16(\mathrm{~d}, 2 \mathrm{H}$, $\left.J=7,8 \mathrm{~Hz}, \mathrm{H}_{\text {aromático }}\right) ; 5,92-5,77\left(\mathrm{~m}, 1 \mathrm{H}, \mathrm{C} \underline{\mathrm{H}}=\mathrm{CH}_{2}\right) ; 5,23-5,14(\mathrm{~m}$, $\left.2 \mathrm{H}, \mathrm{CH}=\mathrm{CH}_{2}\right) ; 4,74(\mathrm{t}, 1 \mathrm{H}, J=6,6 \mathrm{~Hz}, \mathrm{C} \underline{\mathrm{HOH}}) ; 2,55-2,49(\mathrm{~m}, 2 \mathrm{H}$, $\left.\mathrm{CHCH}_{2}\right) ; 2,38\left(\mathrm{~s}, 3 \mathrm{H}, \mathrm{CH}_{3}\right) ; 1,79$ (sl, $\left.1 \mathrm{H}, \mathrm{OH}\right) . \mathrm{RMN}{ }^{13} \mathrm{C}(75 \mathrm{MHz}$, $\left.\mathrm{CDCl}_{3}\right) \delta 141,2 ; 137,5 ; 134,9 ; 129,0 ; 125,7 ; 118,6 ; 73,5 ; 44,1 ; 21,4$.

1-(4-Metoxifenil)but-3-en-1-ol (5d): Obtido $75 \mathrm{mg}(84 \%)$; $\mathrm{RMN}{ }^{1} \mathrm{H}\left(300 \mathrm{MHz}, \mathrm{CDCl}_{3}\right) \delta 7,78\left(\mathrm{~d}, 2 \mathrm{H}, J=9,0 \mathrm{~Hz}, \mathrm{H}_{\text {aromático }}\right)$; $7,38\left(\mathrm{~d}, 2 \mathrm{H}, J=9,0 \mathrm{~Hz}, \mathrm{H}_{\text {aromático }}\right) ; 6,29$ (ddt, $1 \mathrm{H}, J=16,8 ; 9,9$ e 6,6 $\left.\mathrm{Hz}, \mathrm{C} \underline{\mathrm{H}}=\mathrm{CH}_{2}\right) ; 5,68-5,58\left(\mathrm{~m}, 2 \mathrm{H}, \mathrm{CH}=\mathrm{CH}_{2}\right) ; 5,17(\mathrm{t}, 1 \mathrm{H}, J=6,6 \mathrm{~Hz}$, $\mathrm{C} \underline{\mathrm{HOH}}) ; 4,29$ (s, 3H, OMe); 3,02-2,95 (m, 2H, $\left.\mathrm{CHCH}_{2}\right) ; 2,50$ (sl, $1 \mathrm{H}, \mathrm{OH}) . \mathrm{RMN}{ }^{13} \mathrm{C}\left(75 \mathrm{MHz}, \mathrm{CDCl}_{3}\right) \delta 159,3 ; 136,4 ; 134,9 ; 127,4$; 116,$5 ; 114,1 ; 74,3 ; 55,6 ; 44,1$.

1-(3-Metoxifenil)but-3-en-1-ol (5e): Obtido $78 \mathrm{mg}(88 \%)$; RMN ${ }^{1} \mathrm{H}\left(300 \mathrm{MHz}, \mathrm{CDCl}_{3}\right) \delta 7,30\left(\mathrm{dd}, 1 \mathrm{H}, J=8,1\right.$ e 7,8 Hz, $\left.\mathrm{H}_{\text {aromático }}\right)$; 6,98-6,94 (m, 2H, $\left.\mathrm{H}_{\text {aromático }}\right), 6,84$ (ddd, $1 \mathrm{H}, J=8,1 ; 2,7$ e $1,2 \mathrm{~Hz}$, $\mathrm{H}_{\text {aromático }}$ ); 5,85 (ddt, $1 \mathrm{H}, J=17,1 ; 10,2$ e 7,5 Hz, $\left.\mathrm{C} \underline{H}=\mathrm{CH}_{2}\right) ; 5,23-5,15$ $\left(\mathrm{m}, 2 \mathrm{H}, \mathrm{CH}=\mathrm{C}_{2}\right) ; 4,74(\mathrm{dd}, 1 \mathrm{H}, J=7,5$ e $5,4 \mathrm{~Hz}, \mathrm{C} \underline{\mathrm{HOH}}) ; 3,83$ (s, $3 \mathrm{H}, \mathrm{OMe}) ; 2,56-2,50\left(\mathrm{~m}, 2 \mathrm{H}, \mathrm{CHCH}_{2}\right) ; 2,06$ (sl, 1H, OH). RMN ${ }^{13} \mathrm{C}$ $\left(75 \mathrm{MHz}, \mathrm{CDCl}_{3}\right) \delta 159,9 ; 145,9 ; 134,7 ; 129,7 ; 118,5 ; 118,4 ; 113,2$; 111,$6 ; 73,5 ; 55,5 ; 44,0$.
1-(2-Metoxifenil)but-3-en-1-ol (5f): Obtido $76 \mathrm{mg}$ (86\%); RMN ${ }^{1} \mathrm{H}\left(300 \mathrm{MHz}, \mathrm{CDCl}_{3}\right) \delta 7,32\left(\mathrm{dd}, 1 \mathrm{H}, J=7,5\right.$ e 1,8 Hz, $\left.\mathrm{H}_{\text {aromático }}\right)$; 7,27 (td, $1 \mathrm{H}, J=7,5$ e 1,8 Hz, $\left.\mathrm{H}_{\text {aromático }}\right) ; 6,94$ (td, $1 \mathrm{H}, J=8,4$ e 1,2 $\left.\mathrm{Hz}, \mathrm{H}_{\text {aromático }}\right) ; 6,86\left(\mathrm{~d}, 1 \mathrm{H}, J=8,4 \mathrm{~Hz}, \mathrm{H}_{\text {aromático }}\right) ; 5,84$ (ddt, $J=17,1$;

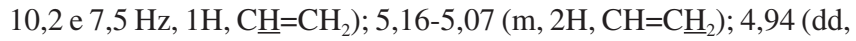
$J=8,1$ e $5,1 \mathrm{~Hz}, 1 \mathrm{H}, \mathrm{C} \underline{\mathrm{HOH}}) ; 3,83$ (s, 3H, OMe); 2,63-2,42 (m, 2H, $\left.\mathrm{CHCH}_{2}\right) ; 2,40(\mathrm{sl}, 1 \mathrm{H}, \mathrm{OH}) . \mathrm{RMN}^{13} \mathrm{C}\left(75 \mathrm{MHz}, \mathrm{CDCl}_{3}\right) \delta 156,2$; 136,$1 ; 131,6 ; 128,1 ; 126,6 ; 120,5 ; 117,4 ; 110,2 ; 69,4 ; 55,1 ; 41,7$.

1-(4-Nitrofenil)but-3-en-1-ol (5g): Obtido 76 mg (79\%); RMN ${ }^{1} \mathrm{H}\left(300 \mathrm{MHz}, \mathrm{CDCl}_{3}\right) \delta 8,20\left(\mathrm{~d}, 2 \mathrm{H}, J=8,7 \mathrm{~Hz}, \mathrm{H}_{\text {aromático }}\right) ; 7,53$ (d, $\left.2 \mathrm{H}, J=8,7 \mathrm{~Hz}, \mathrm{H}_{\text {aromático }}\right) ; 5,85-5,71\left(\mathrm{~m}, 1 \mathrm{H}, \mathrm{C} \underline{\mathrm{H}}=\mathrm{CH}_{2}\right) ; 5,22-5,16$ (m, $\left.2 \mathrm{H}, \mathrm{CH}=\mathrm{CH}_{2}\right) ; 4,86(\mathrm{dd}, 1 \mathrm{H}, J=7,8$ e $4,5 \mathrm{~Hz}, \mathrm{CHOH}) ; 2,61-2,39$ $\left(\mathrm{m}, 2 \mathrm{H}, \mathrm{CHC}_{2}\right) ; 2,15(\mathrm{sl}, 1 \mathrm{H}, \mathrm{OH}) . \mathrm{RMN}^{13} \mathrm{C}\left(75 \mathrm{MHz}, \mathrm{CDCl}_{3}\right) \delta$ 151,$1 ; 147,1 ; 133,1 ; 126,5 ; 123,5 ; 119,5 ; 72,1 ; 43,8$.

1-(3-Nitrofenil)but-3-en-1-ol (5h): Obtido $72 \mathrm{mg}$ (75\%); RMN ${ }^{1} \mathrm{H}\left(300 \mathrm{MHz}, \mathrm{CDCl}_{3}\right) \delta 8,24$ (t, $\left.1 \mathrm{H}, J=1,5 \mathrm{~Hz}, \mathrm{H}_{\text {aromático }}\right) ; 8,13$ (ddd, $1 \mathrm{H}, J=8,1 ; 2,1$ e $\left.0,9 \mathrm{~Hz}, \mathrm{H}_{\mathrm{Ar}}\right) ; 7,69\left(\mathrm{~d}, 1 \mathrm{H}, J=8,1 \mathrm{~Hz}, \mathrm{H}_{\text {aromático }}\right) ; 7,53$ (t, $\left.1 \mathrm{H}, J=8,1 \mathrm{~Hz}, \mathrm{H}_{\text {aromático }}\right) ; 5,86-5,71\left(\mathrm{~m}, 1 \mathrm{H}, \mathrm{C} \underline{\mathrm{H}}=\mathrm{CH}_{2}\right) ; 5,22-5,14$ (m, $\left.2 \mathrm{H}, \mathrm{CH}=\mathrm{C}_{2}\right) ; 4,86$ (dd, $1 \mathrm{H}, J=8,1$ e $\left.5,1 \mathrm{~Hz}, \mathrm{C} \underline{\mathrm{HOH}}\right) ; 2,62-2,42$ $\left(\mathrm{m}, 2 \mathrm{H}, \mathrm{CHC}_{2}\right) ; 2,17$ (sl, $\left.1 \mathrm{H}, \mathrm{OH}\right) . \mathrm{RMN}^{13} \mathrm{C}\left(75 \mathrm{MHz}, \mathrm{CDCl}_{3}\right) \delta$ 147,$8 ; 145,4 ; 132,7 ; 131,5 ; 128,8 ; 121,9 ; 120.3 ; 119,1 ; 71,5 ; 43,4$.

1-(2-Nitrofenil)but-3-en-1-ol (5i): Obtido $87 \mathrm{mg}$ (90\%); RMN ${ }^{1} \mathrm{H}\left(300 \mathrm{MHz}, \mathrm{CDCl}_{3}\right) \delta 7,93\left(\mathrm{dd}, 1 \mathrm{H}, J=8,1\right.$ e $\left.1,2 \mathrm{~Hz}, \mathrm{H}_{\text {aromático }}\right)$; $7,84\left(\mathrm{dd}, 1 \mathrm{H}, J=8,1\right.$ e $\left.1,5 \mathrm{~Hz}, \mathrm{H}_{\text {aromático }}\right) ; 7,68$ (td, $1 \mathrm{H}, J=8,1$ e 1,2 $\left.\mathrm{Hz}, \mathrm{H}_{\text {aromático }}\right) ; 7,43$ (td, $1 \mathrm{H}, J=8,1$ e 1,2 Hz, $\left.\mathrm{H}_{\text {aromático }}\right) ; 5,97-5,83$ (m, $\left.1 \mathrm{H}, \mathrm{C} \underline{\mathrm{H}}=\mathrm{CH}_{2}\right) ; 5,32(\mathrm{dd}, 1 \mathrm{H}, J=8,4$ e $3,6 \mathrm{~Hz}, \mathrm{CHOH}) ; 5,24-5,18$ $\left(\mathrm{m}, 2 \mathrm{H}, \mathrm{CH}=\mathrm{CH}_{2}\right), 2,76-2,67\left(\mathrm{~m}, 1 \mathrm{H}, \mathrm{CHC}_{2}\right), 2,47-2,36(\mathrm{~m}, 2 \mathrm{H}$, $\mathrm{CHCH}_{2}$ e $\left.\mathrm{OH}\right) . \mathrm{RMN}{ }^{13} \mathrm{C}\left(75 \mathrm{MHz}, \mathrm{CDCl}_{3}\right) \delta 148,0 ; 139,6 ; 134,3$; 133,$8 ; 128,5 ; 128,4 ; 124,7 ; 119,4 ; 68,7 ; 43,2$.

1-(2-Fluorofenil)but-3-en-1-ol (5j): Obtido 49 mg (59\%); RMN ${ }^{1} \mathrm{H}\left(300 \mathrm{MHz}, \mathrm{CDCl}_{3}\right) \delta 7,42\left(\mathrm{t}, 1 \mathrm{H}, J=7,6 \mathrm{~Hz}, \mathrm{H}_{\text {aromática }}\right) ; 7,21-7,14$ (m, 1H, $\left.\mathrm{H}_{\text {aromática }}\right) ; 7,08\left(\mathrm{t}, 1 \mathrm{H}, J=7,6 \mathrm{~Hz}, \mathrm{H}_{\text {aromático }}\right) ; 6,96-6,91(\mathrm{~m}, 1 \mathrm{H}$, $\left.\mathrm{H}_{\text {aromático }}\right) ; 5,81-5,70\left(\mathrm{~m}, 1 \mathrm{H}, \mathrm{C} \underline{\mathrm{H}}=\mathrm{CH}_{2}\right) ; 5,12-5,07$ (m, 2H, $\left.\mathrm{CH}=\mathrm{C}_{2}\right)$; 5,02 (dd, $1 \mathrm{H}, J=7,6$ e 4,4 Hz, $\mathrm{C} \underline{\mathrm{HOH}}) ; 2,56-2,39$ (m, 2H, $\left.\mathrm{CHC}_{2}\right)$; $1,82(\mathrm{sl}, 1 \mathrm{H}, \mathrm{OH}) . \mathrm{RMN}^{13} \mathrm{C}\left(75 \mathrm{MHz}, \mathrm{CDCl}_{3}\right) \delta 160,0\left(\mathrm{~d}, J_{C-F}=183,5\right.$ $\mathrm{Hz}) ; 134,3 ; 131,1\left(\mathrm{~d}, J_{C-F}=9,9 \mathrm{~Hz}\right) ; 129,1\left(\mathrm{~d}, J_{C-F}=6,4 \mathrm{~Hz}\right) ; 127,5$; 119,$0 ; 115,5\left(\mathrm{~d}, J_{C-F}=16.3 \mathrm{~Hz}\right) ; 67,5 ; 42,9$.

1-(4-Fluorofenil)but-3-en-1-ol (5k): Obtido $33 \mathrm{mg}$ (40\%); RMN ${ }^{1} \mathrm{H}\left(300 \mathrm{MHz}, \mathrm{CDCl}_{3}\right) \delta 7,29-7,24\left(\mathrm{~m}, 2 \mathrm{H}, \mathrm{H}_{\text {aromática }}\right) ; 6,97$ (t, 2H, J $\left.=8,4 \mathrm{~Hz}, \mathrm{H}_{\text {aromática }}\right) ; 5,80-5,66\left(\mathrm{~m}, 1 \mathrm{H}, \mathrm{C} \underline{\mathrm{H}}=\mathrm{CH}_{2}\right) ; 5,13-5,08(\mathrm{~m}, 2 \mathrm{H}$, $\left.\mathrm{CH}=\mathrm{C}_{2}\right) ; 4,67(\mathrm{dd}, 1 \mathrm{H}, J=7,2$ e $5,7 \mathrm{~Hz}, \mathrm{C} \underline{\mathrm{HOH}}) ; 2,51-2,39(\mathrm{~m}, 2 \mathrm{H}$, $\left.\mathrm{CHCH}_{2}\right) ; 1,86(\mathrm{sl}, 1 \mathrm{H}, \mathrm{OH}) . \mathrm{RMN}^{13} \mathrm{C}\left(75 \mathrm{MHz}, \mathrm{CDCl}_{3}\right) \delta 162,5(\mathrm{~d}$, $\left.J_{C-F}=182,9 \mathrm{~Hz}\right) ; 139,9 ; 134,4 ; 127,7\left(\mathrm{~d}, J_{C-F}=6,4 \mathrm{~Hz}\right) ; 119,0 ; 115.5$ $\left(\mathrm{d}, J_{C-F}=15,7 \mathrm{~Hz}\right) ; 72,9 ; 44,3$.

1-(4-Clorofenil)but-3-en-1-ol (5l): Obtido $81 \mathrm{mg}$ (89\%); RMN ${ }^{1} \mathrm{H}\left(300 \mathrm{MHz}, \mathrm{CDCl}_{3}\right) \delta 7,27-7,18\left(\mathrm{~m}, 4 \mathrm{H}, \mathrm{H}_{\text {aromático }}\right) ; 5,78-5,61(\mathrm{~m}$, $\left.1 \mathrm{H}, \mathrm{C} \underline{\mathrm{H}}=\mathrm{CH}_{2}\right) ; 5,13-5,04\left(\mathrm{~m}, 2 \mathrm{H}, \mathrm{CH}=\mathrm{CH}_{2}\right) ; 4,66(\mathrm{dd}, J=7,5$ e 5,4 $\mathrm{Hz}, \mathrm{CHOH}) ; 2,48-2,31$ (m, $\left.2 \mathrm{H}, \mathrm{CHCH}_{2}\right) ; 2,04$ (sl, 1H, OH). RMN ${ }^{13} \mathrm{C}$ $\left(75 \mathrm{MHz}, \mathrm{CDCl}_{3}\right) \delta 142,2 ; 133,9 ; 131,0 ; 128,4 ; 127,1 ; 118,8 ; 72,5$; 43,8 .

1-(4-Bromofenil)but-3-en-1-ol (5m): Obtido $82 \mathrm{mg}$ (73\%); $\mathrm{RMN}^{1} \mathrm{H}\left(300 \mathrm{MHz}, \mathrm{CDCl}_{3}\right) \delta 7,42\left(\mathrm{~d}, 2 \mathrm{H}, J=8,7 \mathrm{~Hz}, \mathrm{H}_{\text {aromático }}\right) ; 7,18$ (d, 2H, $\left.J=8,7 \mathrm{~Hz}, \mathrm{H}_{\text {aromático }}\right) ; 5,79-5,63\left(\mathrm{~m}, 1 \mathrm{H}, \mathrm{C} \underline{\mathrm{H}}=\mathrm{CH}_{2}\right) ; 5,14-5,05$ (m, $\left.2 \mathrm{H}, \mathrm{CH}=\mathrm{C}_{2}\right) ; 4,65$ (dd, $1 \mathrm{H}, J=7,8$ e $5,4 \mathrm{~Hz}, \mathrm{C} \underline{\mathrm{HOH}}$ ); 2,49-2,33 $\left(\mathrm{m}, 2 \mathrm{H}, \mathrm{CHC}_{2}\right) ; 1,99(\mathrm{sl}, 1 \mathrm{H}, \mathrm{OH}) . \mathrm{RMN}{ }^{13} \mathrm{C}\left(75 \mathrm{MHz}, \mathrm{CDCl}_{3}\right) \delta$ 143,$1 ; 134,3 ; 131,8 ; 127,9 ; 121,6 ; 119,3 ; 72,9 ; 44,2$. 
1-(5-Bromo-2-metoxifenil)but-3-em-1-ol (5n): Obtido 104 $\mathrm{mg}(82 \%)$; $\mathrm{RMN}^{1} \mathrm{H}\left(400 \mathrm{MHz}, \mathrm{CDCl}_{3}\right) \delta 7,49(\mathrm{~d}, 1 \mathrm{H}, J=2,4 \mathrm{~Hz}$, $\left.\mathrm{H}_{\text {aromático }}\right) ; 7,33$ (dd, $1 \mathrm{H}, J=8,4$ e 2,4 Hz, $\left.\mathrm{H}_{\text {aromático }}\right) ; 6,74$ (d, 1H, $J=$ $\left.8,4 \mathrm{~Hz}, \mathrm{H}_{\text {aromático }}\right) ; 5,88-5,78\left(\mathrm{~m}, 1 \mathrm{H}, \mathrm{CH}=\mathrm{CH}_{2}\right) ; 5,17-5,12(\mathrm{~m}, 2 \mathrm{H}$, $\left.\mathrm{CH}=\underline{\mathrm{C}}_{2}\right) ; 4,94$ (dd, $1 \mathrm{H}, J=8,0$ e 4,4 Hz, $\left.\mathrm{CHOH}\right) ; 3,83$ (s, 3H, OMe);

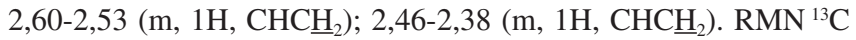
$\left(100 \mathrm{MHz}, \mathrm{CDCl}_{3}\right) \delta 155,5 ; 134,8 ; 134,4 ; 131,0 ; 129,8 ; 118,4 ; 113,4$; 112,$3 ; 68,7 ; 55,8 ; 42,0$.

1-(Furan-2-ilbut-3-en-1-ol (5o): Obtido 42 mg (61\%); RMN ${ }^{1} \mathrm{H}\left(300 \mathrm{MHz}, \mathrm{CDCl}_{3}\right) \delta 7,39\left(\mathrm{dd}, 1 \mathrm{H}, J=1,8\right.$ e $\left.0,9 \mathrm{~Hz}, \mathrm{H}_{\text {heteroaromático }}\right)$; $6,33\left(\mathrm{dd}, 1 \mathrm{H}, J=2,1\right.$ e $\left.1,8 \mathrm{~Hz}, \mathrm{H}_{\text {heteroaromático }}\right) ; 6,26$ (dd, $1 \mathrm{H}, J=$ 2,1 e $\left.0,9 \mathrm{~Hz}, \mathrm{H}_{\text {heteroaromático }}\right) ; 5,82(\mathrm{ddt}, 1 \mathrm{H}, J=17,1 ; 10,2$ e $6,9 \mathrm{~Hz}$, $\left.\mathrm{C} \underline{\mathrm{H}}=\mathrm{CH}_{2}\right) ; 5,23-5,12\left(\mathrm{~m}, 2 \mathrm{H}, \mathrm{CH}=\mathrm{C}_{2}\right) ; 4,75(\mathrm{dd}, 1 \mathrm{H}, J=6,6$ e 6,3 $\mathrm{Hz}, \mathrm{C} \underline{\mathrm{HOH}}) ; 2,66-2,60$ (m, 2H, $\left.\mathrm{CHCH}_{2}\right) ; 2,15$ (sl, 1H, OH). RMN ${ }^{13} \mathrm{C}$ $\left(75 \mathrm{MHz}, \mathrm{CDCl}_{3}\right) \delta 155,9 ; 141,9 ; 133,6 ; 118,6 ; 110,1 ; 106,0 ; 66,9$; 40,0 .

(E)-1-Fenilhexa-1,5-dien-3-ol (5p): Obtido $80 \mathrm{mg}$ (92\%); $\mathrm{RMN}^{1} \mathrm{H}\left(300 \mathrm{MHz}, \mathrm{CDCl}_{3}\right) \delta 7,41-7,20\left(\mathrm{~m}, 5 \mathrm{H}, \mathrm{H}_{\text {aromático }}\right) ; 6,62$ (dd, $1 \mathrm{H}, J=15,9$ e $1,2 \mathrm{~Hz}, \mathrm{PhC} \underline{\mathrm{H}}=\mathrm{CH}) ; 6,23(\mathrm{dd}, 1 \mathrm{H}, J=15,9$ e 6,3 $\mathrm{Hz}, \mathrm{PhCH}=\mathrm{CH}) ; 5,86\left(\mathrm{ddt}, 1 \mathrm{H}, J=17,1 ; 10,2\right.$ e $\left.6,9 \mathrm{~Hz}, \mathrm{C} \underline{\mathrm{H}}=\mathrm{CH}_{2}\right)$; 5,22-5,14 (m, 2H, $\left.\mathrm{CH}=\mathrm{CH}_{2}\right)$; 4,39-4,33 (m, 1H, CㅂH); 2,50-2,34 $\left(\mathrm{m}, 2 \mathrm{H}, \mathrm{CHC}_{2}\right) ; 1,78(\mathrm{sl}, 1 \mathrm{H}, \mathrm{OH}) . \mathrm{RMN}^{13} \mathrm{C}\left(75 \mathrm{MHz}, \mathrm{CDCl}_{3}\right) \delta$ 136,$5 ; 133,9 ; 131,4 ; 130,2 ; 128,4 ; 127,5 ; 126,3 ; 118,3 ; 71,6 ; 41,8$.

2-Metilhex-5-en-3-ol (5q): Obtido $34 \mathrm{mg}$ (59\%); RMN ${ }^{1} \mathrm{H}$ $\left(300 \mathrm{MHz}, \mathrm{CDCl}_{3}\right.$ ) $\delta 5,82$ (dddd, $J=17,1 ; 10,8 ; 8,1$ e $6,3 \mathrm{~Hz}, 1 \mathrm{H}$, $\left.\mathrm{C} \underline{\mathrm{H}}=\mathrm{CH}_{2}\right) ; 5,17-5,10\left(\mathrm{~m}, 2 \mathrm{H}, \mathrm{CH}=\mathrm{CH}_{2}\right) ; 3,42-3,36(\mathrm{~m}, 1 \mathrm{H}, \mathrm{CHOH})$; 2,36-2,26 (m, 2H, $\left.\mathrm{CHCH}_{2}\right) ; 2,16-2,05\left(\mathrm{~m}, 1 \mathrm{H}, \mathrm{CH}_{3} \mathrm{CH}\right)$; 1,67 (sl, 1H, $\mathrm{OH}) ; 0,93\left(\mathrm{~d}, 6 \mathrm{H}, J=3,9 \mathrm{~Hz}, \mathrm{CH}_{3} \mathrm{CH}\right) . \mathrm{RMN}^{13} \mathrm{C}\left(75 \mathrm{MHz}, \mathrm{CDCl}_{3}\right)$ $\delta 135,8,118,3,75,7,39,2,33,4,19,1,17,9$.

Non-1-en-4-ol (5r): Obtido $44 \mathrm{mg}(62 \%)$; $\mathrm{RMN}^{1} \mathrm{H}(300 \mathrm{MHz}$, $\left.\mathrm{CDCl}_{3}\right) \delta 5,88-5,75\left(\mathrm{~m}, 1 \mathrm{H}, \mathrm{CH}=\mathrm{CH}_{2}\right) ; 5,28-5,09\left(\mathrm{~m}, 2 \mathrm{H}, \mathrm{CH}=\mathrm{CH}_{2}\right)$; 3,69-3,58 (m, 1H, C프); 2,35-2,24 (m, 2H, $\left.\mathrm{CHC}_{2}\right) ; 2,18-2,07$ (m, $2 \mathrm{H}, \mathrm{C} \underline{\mathrm{HOH}}$ ); 1,65 (sl, 1H, OH); 150-1,24 (m, 6H, $\mathrm{CH}_{3} \underline{\mathrm{H}}_{2} \mathrm{CH}_{2} \mathrm{CH}_{2}$ ); 0,87 (t, $\left.6 \mathrm{H}, J=6,3 \mathrm{~Hz}, \mathrm{C}_{3} \mathrm{CH}_{2}\right) . \mathrm{RMN}^{13} \mathrm{C}\left(75 \mathrm{MHz}, \mathrm{CDCl}_{3}\right) \delta 134,8$; 118,$0 ; 70,6 ; 41,9 ; 36,7 ; 31,8 ; 25,3 ; 22,7,14,0$.

Ácido 4-(1-hidroxibut-3-enil)benzoico (5s): Obtido $72 \mathrm{mg}$ (75\%); $\mathrm{RMN}^{1} \mathrm{H}\left(400 \mathrm{MHz}, \mathrm{CDCl}_{3}\right) \delta 8,10(\mathrm{~d}, 2 \mathrm{H}, J=7,6 \mathrm{~Hz}$, $\left.\mathrm{H}_{\text {aromático }}\right) ; 7,48\left(\mathrm{~d}, 2 \mathrm{H}, J=7,6 \mathrm{~Hz}, \mathrm{H}_{\text {aromático }}\right) ; 5,86-5,76(\mathrm{~m}, 1 \mathrm{H}$, $\left.\mathrm{C} \underline{\mathrm{H}}=\mathrm{CH}_{2}\right) ; 5,21-5,17\left(\mathrm{~m}, 2 \mathrm{H}, \mathrm{CH}=\mathrm{C}_{2}\right) ; 4,84(\mathrm{dd}, 1 \mathrm{H}, J=8,0$ e 4,8 $\mathrm{Hz}, \mathrm{CHOH}) ; 2,61-2,45$ (m, 2H, $\left.\mathrm{CHC}_{2}\right) ; 1,26$ (sl, 1H, OH). RMN ${ }^{13} \mathrm{C}\left(100 \mathrm{MHz}, \mathrm{CDCl}_{3}\right) \delta 172,0 ; 150,1 ; 133,9 ; 130,6 ; 128,7 ; 126,1$; 119,$4 ; 73,0 ; 44,1$.

Metil 4-(1-hidroxibut-3-enil)benzoato (5t): Obtido $79 \mathrm{mg}$ (77\%); $\mathrm{RMN}^{1} \mathrm{H}\left(400 \mathrm{MHz}, \mathrm{CDCl}_{3}\right) \delta 8,01(\mathrm{~d}, 2 \mathrm{H}, J=7,2 \mathrm{~Hz}$, $\left.\mathrm{H}_{\text {aromático }}\right) ; 7,42\left(\mathrm{~d}, 2 \mathrm{H}, J=7,2 \mathrm{~Hz}, \mathrm{H}_{\text {aromático }}\right) ; 5,84-5,73(\mathrm{~m}, 1 \mathrm{H}$, $\left.\mathrm{C} \underline{\mathrm{H}}=\mathrm{CH}_{2}\right) ; 5,19-5,15\left(\mathrm{~m}, 2 \mathrm{H}, \mathrm{CH}=\mathrm{C}_{2}\right) ; 4,80(\mathrm{dd}, 1 \mathrm{H}, J=7,6$ e 5,2 $\mathrm{Hz}, \mathrm{C} \underline{\mathrm{HOH}}) ; 3,91$ (s, 3H, OMe); 2,56-2,43 (m, 2H, $\left.\mathrm{CHCH}_{2}\right) ; 2,19$ (sl, 1H, OH). RMN ${ }^{13} \mathrm{C}\left(100 \mathrm{MHz}, \mathrm{CDCl}_{3}\right) \delta 167,2 ; 149,2 ; 134,1$; 130,$0 ; 129,5 ; 126,0 ; 119,2 ; 73,0 ; 52,3 ; 44,1$.

4-(1-hidroxibut-3-enil)benzonitrila (5u): Obtido $80 \mathrm{mg}$ (93\%); $\mathrm{RMN}^{1} \mathrm{H}\left(400 \mathrm{MHz}, \mathrm{CDCl}_{3}\right) \delta 7,62\left(\mathrm{~d}, 2 \mathrm{H}, J=8,4 \mathrm{~Hz}, \mathrm{H}_{\text {aromático }}\right) ; 7,46$ (d, $\left.2 \mathrm{H}, J=8,4 \mathrm{~Hz}, \mathrm{H}_{\text {aromático }}\right) ; 5,81-5,72\left(\mathrm{~m}, 1 \mathrm{H}, \mathrm{C} \underline{\mathrm{H}}=\mathrm{CH}_{2}\right) ; 5,18-5,14$ (m, $\left.2 \mathrm{H}, \mathrm{CH}=\mathrm{C}_{2}\right) ; 4,80$ (dd, $1 \mathrm{H}, J=8,4$ e $4,4 \mathrm{~Hz}, \mathrm{CHOH}$ ); 2,56-2,49 (m, $\left.1 \mathrm{H}, \mathrm{C}_{2} \mathrm{CH}=\mathrm{CH}_{2}\right) ; 2,47-2,41\left(\mathrm{~m}, 1 \mathrm{H}, \mathrm{CH}_{2} \mathrm{CH}=\mathrm{CH}_{2}\right) ; 2,27$ (sl, $1 \mathrm{H}, \mathrm{OH}) .{ }^{13} \mathrm{C} \mathrm{NMR}\left(100 \mathrm{MHz}, \mathrm{CDCl}_{3}\right) \delta 149,3 ; 133,1 ; 132,2 ; 128,7$; 119,$7 ; 119,1 ; 111,4 ; 72,6 ; 44,1$. 1-(4-(1-hidroxibut-3-enil)fenil)etanona (5v): Obtido $82 \mathrm{mg}$ (86\%); $\mathrm{RMN}{ }^{1} \mathrm{H}\left(400 \mathrm{MHz}, \mathrm{CDCl}_{3}\right) \delta 7,93(\mathrm{~d}, 2 \mathrm{H}, J=8,0 \mathrm{~Hz}$, $\left.\mathrm{H}_{\text {aromático }}\right) ; 7,44\left(\mathrm{~d}, 2 \mathrm{H}, J=8,0 \mathrm{~Hz}, \mathrm{H}_{\text {aromático }}\right) ; 5,84-5,74(\mathrm{~m}, 1 \mathrm{H}$, $\left.\mathrm{C} \underline{\mathrm{H}}=\mathrm{CH}_{2}\right) ; 5,19-5,14\left(\mathrm{~m}, 2 \mathrm{H}, \mathrm{CH}=\mathrm{CH}_{2}\right) ; 4,81$ (dd, $1 \mathrm{H}, J=8,0$ e 5,2 $\mathrm{Hz}, \mathrm{C} \underline{\mathrm{HOH}}) ; 2,59$ (s, 3H, $\left.\mathrm{CH}_{3}\right) ; 2,58-2,43\left(\mathrm{~m}, 2 \mathrm{H}, \mathrm{C}_{2} \mathrm{CH}=\mathrm{CH}_{2}\right) ; 2,21$ (sl, 1H, OH). RMN ${ }^{13} \mathrm{C}\left(100 \mathrm{MHz}, \mathrm{CDCl}_{3}\right) \delta 198,1 ; 149,4 ; 133,6$; 128,$8 ; 126,2 ; 119,3 ; 72,9 ; 44,1 ; 26,9$.

1-(3-((tert-butildimetilsilil)oxi)-4-metoxifenil)but-3-en-1-ol (5w): Obtido 249,6 mg (81\%); $\mathrm{RMN}^{1} \mathrm{H}\left(400 \mathrm{MHz}, \mathrm{CDCl}_{3}\right) \delta 6,92$ (s, $\left.1 \mathrm{H}, \mathrm{H}_{\text {aromático }}\right) ; 6,73\left(\mathrm{sl}, 2 \mathrm{H}, \mathrm{H}_{\text {aromático }}\right) ; 5,79-5,69\left(\mathrm{~m}, 1 \mathrm{H}, \mathrm{C} \underline{\mathrm{H}}=\mathrm{CH}_{2}\right)$; $5,14(\mathrm{~d}, 1 \mathrm{H}, J=4 \mathrm{~Hz}, \mathrm{CHOH}) ; 5,00-4,93\left(\mathrm{~m}, 2 \mathrm{H}, \mathrm{CH}=\mathrm{CH}_{2}\right) ; 4,50-4,46$ (m, 1H, CHOH); 3,70 (s, 3H, OMe); 2,40-2, 27 (m, 2H, $\mathrm{CH}_{2} \mathrm{CH}=\mathrm{CH}_{2}$ ); 0,93 (s, 9H, $\left.\mathrm{CH}_{3}\right) ; 0,09$ (s, 6H, $\left.\mathrm{CH}_{3}\right) ; \mathrm{RMN}{ }^{13} \mathrm{C}\left(100 \mathrm{MHz}, \mathrm{CDCl}_{3}\right)$ $\delta 150,0 ; 142,9 ; 139,5 ; 135,8 ; 119,7 ; 118,0 ; 116,4 ; 110,1 ; 72,1 ; 55,3$; 43,$8 ; 25,6 ; 18,1 ;-4,8$.

\section{RESULTADOS E DISCUSSÃO}

A primeira etapa do trabalho consistiu na síntese das aril-amidoximas (2a-h) a partir da reação das respectivas aril-nitrilas (1a-h) com cloridato de hidroxilamina, carbonato de sódio em meio hidroetanólico sob irradiação de ultrassom. ${ }^{23}$ Os resultados estão sumarizados na Tabela 1.

De acordo com a Tabela 1, quando foram utilizadas nitrilas aromáticas contendo o grupo metila no anel, foi observado que dependendo da posição deste grupo (orto-, meta-, para-) houve um aumento no rendimento e uma diminuição no tempo reacional (Tabela 1, entradas 2, 3 e 4). Uma provável explicação pode ser atribuída ao impedimento estérico causado pelo grupo metila, o que dificultaria a aproximação do nucleófilo $\left(\mathrm{NH}_{2} \mathrm{OH}\right)$ ao carbono do grupo nitrila. Quando nitrilas contendo grupos retiradores de densidade eletrônica foram utilizadas, as respectivas amidoximas $\mathbf{2 e}, \mathbf{2} \mathbf{f}$ e $\mathbf{2} \mathbf{g}$ foram obtidas em bons rendimentos (Tabela 1, entradas 5, 6, e 8). Adicionalmente, quando uma nitrila heteroaromática foi utilizada, a amidoxima correspondente $(\mathbf{2 g})$ foi obtida em um rendimento de $81 \%$ após $30 \mathrm{~min}$ (Tabela 1, entrada 7).

Uma vez que o aliltrifluoroborato de potássio (3) foi obtido comercialmente, foi iniciado o estudo da viabilidade da aplicação das amidoximas como agentes de transferência de fase em reações de alilação. Desse modo, a primeira variável estudada foi a quantidade de benzamidoxima (2a) a ser empregada nesta reação. Para isso, o 2-naftaldeído (4a), a benzamidoxima (2a) e o aliltrifluoroborato de potássio (3), utilizados como substratos modelo, foram tratados à temperatura ambiente em um sistema bifásico $\mathrm{H}_{2} \mathrm{O}: \mathrm{CH}_{2} \mathrm{Cl}_{2}$ na proporção de 1:1. Todos os experimentos foram acompanhados por cromatografia de camada delgada (CCD). Os resultados estão descritos na Tabela 2 .

De acordo com a Tabela 2, quando a reação foi conduzida sem a presença da benzamixodima (2a) não houve o consumo completo dos reagentes de partida mesmo após um intervalo de tempo de $240 \mathrm{~min}$ (Tabela 2, entrada 7). Contudo, nos casos em que foi utilizada a benzamidoxima (2a) a reação foi completa (Tabela 2, entradas 1-6). É interessante notar que a redução da quantidade de benzamidoxima (2a) de 100 para $5 \mathrm{mmol} \%$ levou ao produto desejado, 5a, em um rendimento de $90 \%$ após 30 minutos (Tabela 2, entrada 6), demonstrando a capacidade da benzamidoxima (2a) de atuar possivelmente como um catalisador de transferência de fase. Um baixo rendimento foi observado quando foram utilizados $150 \mathrm{mmol} \%$ de benzamidoxima (2a) (Tabela 2, entrada 1).

Em seguida foi estudado o efeito do solvente na reação, em que diferentes proporções de $\mathrm{H}_{2} \mathrm{O}$ e $\mathrm{CH}_{2} \mathrm{Cl}_{2}$ foram avaliadas. Os resultados estão descritos na Tabela 3. 
Tabela 1. Síntese das aril-amidoximas (2a-h) a partir das aril-nitrilas (1a-h) sob irradiação de ultrassom

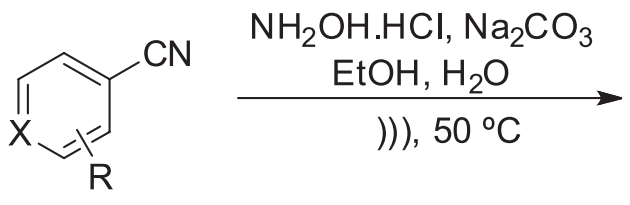<smiles>[R]c1[X]ccc(C(N)=NO)c1</smiles>

1a-h

2a-h

\begin{tabular}{|c|c|c|c|c|c|c|}
\hline Entrada & Nitrila & & Amidoxima & & Tempo (min) & Rendimento $(\%)^{\mathrm{a}}$ \\
\hline 1 & & 1a & & $2 a$ & 20 & 82 \\
\hline 2 & & $1 b$ & & $2 b$ & 45 & 35 \\
\hline 3 & & 1c & & $2 \mathrm{c}$ & 30 & 81 \\
\hline 4 & & 1d & & 2d & 20 & 88 \\
\hline 5 & & 1e & & $2 e$ & 45 & 85 \\
\hline 6 & & 1f & & $2 f$ & 45 & 80 \\
\hline 7 & & $1 \mathrm{~g}$ & & $2 \mathrm{~g}$ & 30 & 81 \\
\hline 8 & & 1h & & $2 \mathrm{~h}$ & 30 & 91 \\
\hline
\end{tabular}

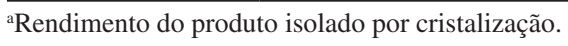

De acordo com a Tabela 3, quando apenas $\mathrm{CH}_{2} \mathrm{Cl}_{2}$ foi utilizado como solvente, nenhum produto de reação foi observado (Tabela 3 , entrada 9), possivelmente devido à baixa solubilidade do alil-trifluoroborato de potássio (3) neste solvente. Quando apenas $\mathrm{H}_{2} \mathrm{O}$ foi utilizada na reação, o produto foi obtido em um rendimento de $69 \%$ (Tabela 3, entrada 1). O melhor resultado foi observado quando um sistema bifásico $\mathrm{H}_{2} \mathrm{O}: \mathrm{CH}_{2} \mathrm{Cl}_{2}$ foi utilizado, sendo que o produto desejado foi obtido em $90 \%$ de rendimento (Tabela 3 , entrada 5).

O estudo do efeito de diferentes aril-amidoximas (2a-h) na reação de alilação também foi realizado e os resultados estão descritos na Tabela 4.

De acordo com a Tabela 4, todas as aril-amidoximas $\mathbf{2 a - h}$ atuaram como catalisadores na reação de alilação, no entanto, o composto 2e levou ao produto desejado em menor tempo reacional (Tabela
4, entrada 5). Diante destes resultados, foi realizado um estudo baseado na espectroscopia na região do Ultravioleta-Vísivel (UV-vis) (Figura 2), a fim de fornecer indícios da ação do composto $\mathbf{2 e}$ como agente de transferência de fase.

De acordo com a Figura 2, o composto $2 \mathbf{e}$ apresentou duas bandas de absorção (em 228 e $253 \mathrm{~nm}$, referente às transições $\mathrm{n} \rightarrow \pi^{*}$ e $\pi \rightarrow \pi^{*}$, respectivamente - Solução A), enquanto que o composto 3 não apresentou absorção na região de estudo (Solução D). Em contrapartida, no espectro de UV-vis da solução aquosa contendo os compostos 3 e 2e (Solução B, Figura 2) houve a supressão da transição $n \rightarrow \pi^{*}$ e deslocamento batocrômico da transição $\pi \rightarrow \pi^{*}$ para a região de $240 \mathrm{~nm}$, implicando em uma possível interação da função química amidoxima do composto 2 e com o potássio do composto 3. Apesar dessa evidencia, a literatura descreve que as bandas de absorção de substâncias ácidas ou básicas podem variar 
Tabela 2. Estudo da influência da quantidade de benzamidoxima (2a) na reação de alilação do 2-naftaldeído (4a) pelo aliltriofluoroborato de potássio (3) em meio bisáfico<smiles>C=CC[Sn+]Br</smiles>

$4 a$
3

$2 \mathrm{a}(\mathrm{mmol} \%)$

100

50

25

10

5

7<smiles>NC(=NO)c1ccccc1</smiles>

t.a.<smiles>C=CCC(O)c1ccc2ccccc2c1</smiles>

$5 a$

\begin{tabular}{lccc}
1 & 150 & 240 & 54 \\
2 & 100 & 120 & 86 \\
3 & 50 & 60 & 84 \\
4 & 25 & 30 & 90 \\
5 & 10 & 30 & 87 \\
6 & 5 & 30 & 90 \\
7 & - & 240 & 40 \\
\hline
\end{tabular}

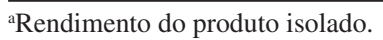

Tabela 3. Estudo da influência da proporção de solvente $\mathrm{H}_{2} \mathrm{O}: \mathrm{CH}_{2} \mathrm{Cl}_{2}$ na reação de alilação do 2-naftaldeido (4a) por aliltrifluoroborato de potássio (3) promovida pela benzamidoxima (2a) à temperatura ambiente

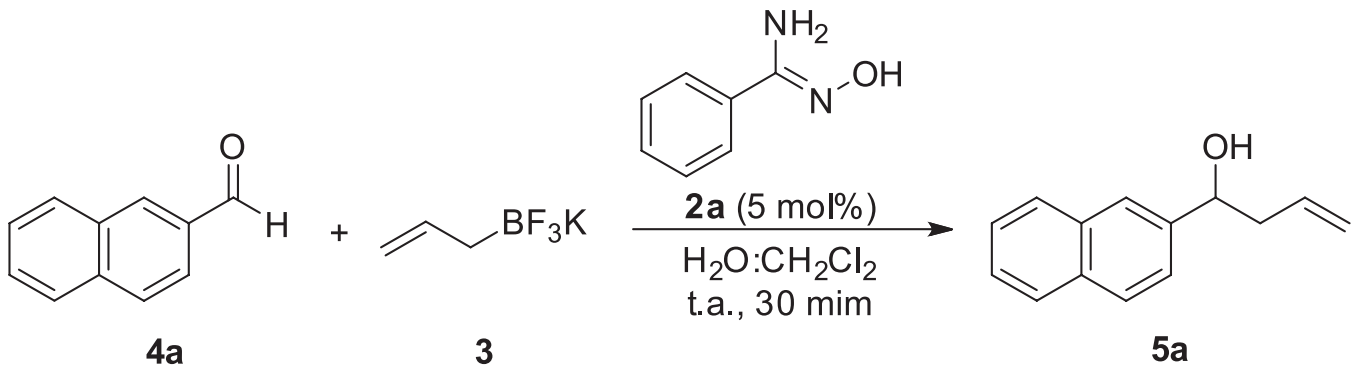

\begin{tabular}{|c|c|c|}
\hline Entrada & $\mathrm{H}_{2} \mathrm{O}: \mathrm{CH}_{2} \mathrm{Cl}_{2}$ & Rendimento $(\%)^{\mathrm{a}}$ \\
\hline 1 & $1: 0$ & 69 \\
\hline 2 & $4: 1$ & 73 \\
\hline 3 & $3: 1$ & 78 \\
\hline 4 & $2: 1$ & 84 \\
\hline 5 & $1: 1$ & 90 \\
\hline 6 & $1: 2$ & 82 \\
\hline 7 & $1: 3$ & 77 \\
\hline 8 & $1: 4$ & 60 \\
\hline 9 & $0: 1$ & 0 \\
\hline
\end{tabular}

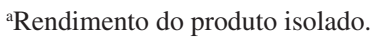

significativamente dependendo do valor do $\mathrm{pH} \cdot{ }^{24}$ Devido a isto, foi realizado o espectro de UV-vis da solução aquosa contendo o composto $2 \mathrm{e}$ e $\mathrm{K}_{2} \mathrm{CO}_{3}$ (Solução $C$, Figura 2), no entanto, não foi observado mudança no perfil de absorção quando comparado ao da solução aquosa do composto 2e (Solução A, Figura 2). Com este resultado preliminar é possível conjecturar que as amidoximas podem atuar, provavelmente, como agente de transferência de fase, porém, outros experimentos são necessários.

Diante dos resultados descritos nas Tabelas 2, 3 e 4, a melhor condição para promover a reação de alilação foi observada quando foi utilizado composto $2 \mathbf{e}\left(5 \mathrm{~mol} \%\right.$ ) em um sistema bifásico $\mathrm{H}_{2} \mathrm{O}: \mathrm{CH}_{2} \mathrm{Cl}_{2}$ (1:1), sendo que o produto desejado foi obtido após $20 \mathrm{~min}$. Esta condição foi então aplicada a outros aldeídos visando a síntese dos álcoois homoalílicos correspondentes. Os resultados estão descritos na Tabela 5.

De acordo com a Tabela 5, o emprego do composto 2e na reação de alilação de diferentes aldeídos levou aos correspondentes álcoois homoalílicos (5a-r) em rendimentos que variaram de moderados a excelentes em intervalos de tempo de 10 a 60 minutos. A alilação de aldeídos contendo grupos doadores de densidade eletrônica no anel, compostos $\mathbf{4 c - f}$, levou aos alcoóis homoalílicos $\mathbf{5 c - f}$ em elevados rendimentos (Tabela 5, entradas 3-6). O mesmo foi constatado quando foram utilizados aldeídos contendo grupos retiradores de densidade eletrônica no anel, compostos 4g-i (Tabela 5, entradas 7-9). Quando foram utilizados substratos halogenados, compostos 4j-n, foi constatado que o aumento da eletronegatividade implicou em uma elevação do tempo reacional, ou seja, para os aldeídos (4-fluor-, 4-cloro- e 4-bromo-)benzaldeído os tempos reacionais foram 60, 20 e 15 minutos, respectivamente (Tabela 5, entradas 10-14). Quando o aldeído heterocíclico 40 foi submetido às condições reacionais de alilação, o álcool homoalílico 50 foi obtido em moderado rendimento (Tabela 5, entrada 15). A reação de alilação de aldeídos alifáticos 4q-r levou à formação dos álcoois homoalílicos $\mathbf{5 q}$-r com rendimentos de 59 a $62 \%$ (Tabela 5, entrada 17 e 18), sendo este baixo rendimento 
Tabela 4. Estudo da influência da aplicação de diferentes aril-amidoximas (2a-h) na reação de alilação do 2-naftaldeído (4a) por aliltrifluoroborato de potássio (3) à temperatura ambiente em um sistema bifásico $\mathrm{H}_{2} \mathrm{O}: \mathrm{CH}_{2} \mathrm{Cl}_{2}(1: 1)$

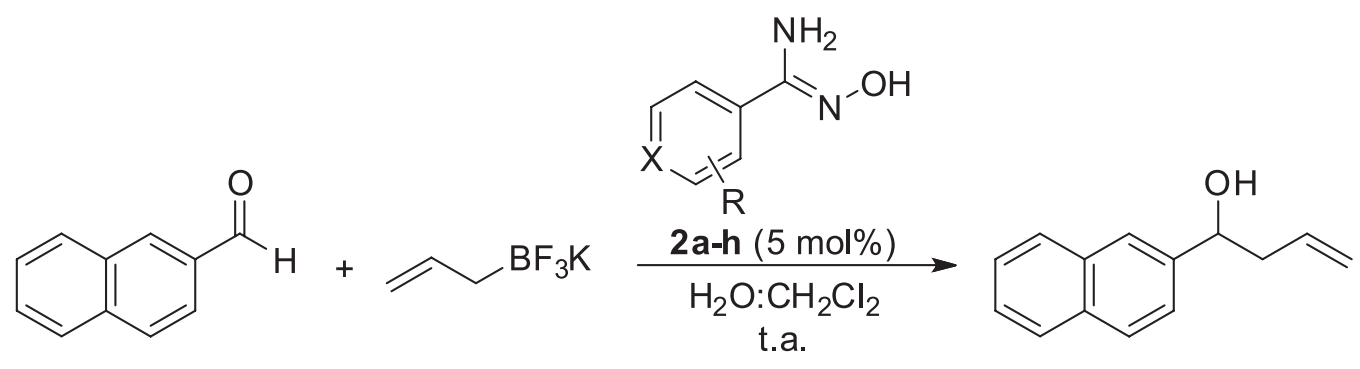

\begin{tabular}{|c|c|c|c|c|}
\hline Entrada & Aril-amidoximas & & Tempo (min) & Rendimento $(\%)^{\mathrm{a}}$ \\
\hline 1 & & $2 a$ & 30 & 90 \\
\hline 2 & & $2 b$ & 25 & 82 \\
\hline 3 & & $2 c$ & 30 & 76 \\
\hline 4 & & $2 d$ & 25 & 73 \\
\hline 5 & & $2 e$ & 20 & 91 \\
\hline 6 & & $2 f$ & 30 & 82 \\
\hline 7 & & $2 \mathrm{~g}$ & 30 & 80 \\
\hline 8 & & $2 \mathrm{~h}$ & 30 & 81 \\
\hline
\end{tabular}

${ }^{\mathrm{a}}$ Rendimento do produto isolado por cristalização.

atribuído, de acordo com a literatura, ${ }^{25}$ a volatilidades dos produtos de partida $\mathbf{5 q - r}$.

Adicionalmente, este método mostrou-se quimiosseletivo, pois foi observado apenas produto de adição 1,2 ao composto $\mathbf{4 p}$, em bom rendimento após 25 minutos (Tabela 5, entrada 11). Devido a esse resultado foi avaliada a quimiosseletividade da reação de alilação utilizando aldeídos contendo outras funcionalidades mais reativas quando comparadas aos exemplos da Tabela 5. Os resultados estão sumarizados na Tabela 6.

De acordo com a Tabela 6 observou-se que a reação foi quimiosseletiva, uma vez que a adição do grupo alil apenas ocorreu na função aldeído apesar dos substratos apresentarem outras funcionalidades. Adicionalmente, em todos os casos os álcoois homoalílicos $\mathbf{5} \mathbf{s}-\mathbf{v}$ foram obtidos com rendimentos que variaram de 77 a $93 \%$ após um tempo reacional que variou de 20 a 30 minutos.

Finalmente, para avaliar se o método era seletivo na presença de um substrato contendo um grupo protetor, foi realizada a reação de alilação do composto $\mathbf{4 w}$ empregando tanto a amidoxima $\mathbf{2 e}$ quanto a amberlyst A-15. A amberlyst A-15 foi escolhida, uma vez que o nosso grupo de pesquisa propôs recentemente o seu emprego como promotor heterogêneo da alilação. ${ }^{25}$ Os resultados estão sumarizados na Tabela 7. 


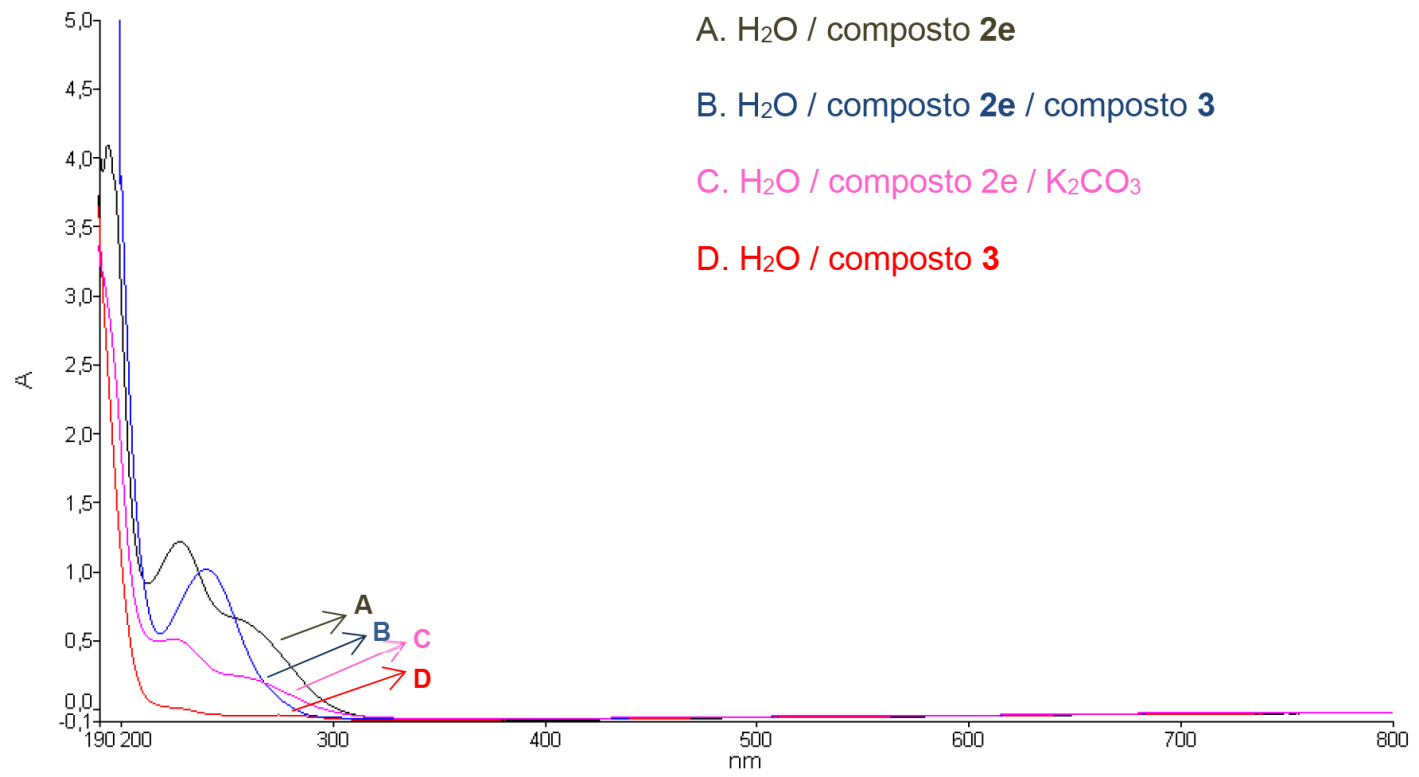

Figura 2. Espectros de absorção na região ultravioleta-visível de (A) Solução aquosa do composto $2 \boldsymbol{e}$; (B) Solução aquosa dos compostos $2 \boldsymbol{e}$ e 3 ; (C) Solução aquosa do composto 2 e e $\mathrm{K}_{2} \mathrm{CO}_{3}$; (D) Solução aquosa do composto 3

Tabela 5. Síntese dos álcoois homoalílicos (5a-r) através da alilação de diversos aldeídos funcionalizados (4a-r) pelo aliltrifluoroborato de potássio (3) catalisada por $\mathbf{2 e}$

(min)


Tabela 5. Síntese dos álcoois homoalílicos (5a-r) através da alilação de diversos aldeídos funcionalizados (4a-r) pelo aliltrifluoroborato de potássio (3) catalisada por $2 \mathbf{e}$ (cont.)

\begin{tabular}{|c|c|c|c|c|c|c|}
\hline Entrada & Aldeído & & Álcool homoalílico & & Tempo (min) & Rendimento $(\%)^{\mathrm{a}}$ \\
\hline 7 & & $4 \mathrm{~g}$ & & $5 \mathrm{~g}$ & 15 & 79 \\
\hline 8 & & $4 h$ & & $5 \mathrm{~h}$ & 20 & 75 \\
\hline 9 & & $4 \mathbf{i}$ & & $5 i$ & 15 & 90 \\
\hline 10 & & $4 \mathbf{j}$ & & $5 \mathbf{j}$ & 30 & $59^{c}$ \\
\hline 11 & & $4 k$ & & $5 \mathbf{k}$ & 60 & $40^{c}$ \\
\hline 12 & & 41 & & 51 & 20 & 89 \\
\hline 13 & & $4 m$ & & $5 \mathrm{~m}$ & 15 & 73 \\
\hline 14 & & $4 n$ & & $5 n$ & 10 & 82 \\
\hline 15 & & 40 & & 50 & 10 & 61 \\
\hline 16 & & $4 p$ & & $5 p$ & 25 & 92 \\
\hline 17 & & $4 q$ & & $5 q$ & 10 & 59 \\
\hline 18 & & $4 r$ & & $5 r$ & 10 & 62 \\
\hline
\end{tabular}

${ }^{a}$ Rendimento do produto isolado. ${ }^{b}$ Rendimento da reação em maior escala e mantendo a mesma quantidade de solvente. Condição experimental: Ao 2-naftaldeído (4a, $234 \mathrm{mg} ; 1,5 \mathrm{mmol})$ em diclorometano $(2 \mathrm{~mL})$ foi adicionada a 4-bromobenzamidoxima (2e, $16,0 \mathrm{mg} ; 0,075 \mathrm{mmol} ; 5 \mathrm{~mol} \%)$, o aliltrifluoroborato

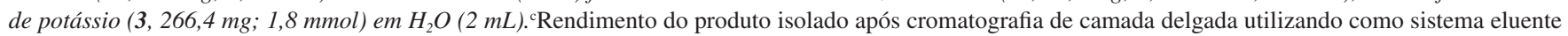
hexano:acetato de etila na proporção $(9,5: 5)$.

De acordo com a Tabela 7, a reação de alilação do composto $\mathbf{4 w}$ empregando o composto 2 e levou à formação exclusiva do álcool homoalílico $\mathbf{5 w}$ quando comparado à reação utilizando a amberlyst A-15, apesar da necessidade de um maior tempo reacional.

\section{CONCLUSÃO}

Neste trabalho foi demonstrada a eficácia da utilização de aril-amidoximas como catalisadores de transferência de fase na reação 
Tabela 6. Síntese dos álcoois homoalílicos (5s-v) através da reação de alilação de diversos aldeídos funcionalizados (4s-v) pelo aliltrifluoroborato de potássio (3) catalisada por $\mathbf{2 e}$

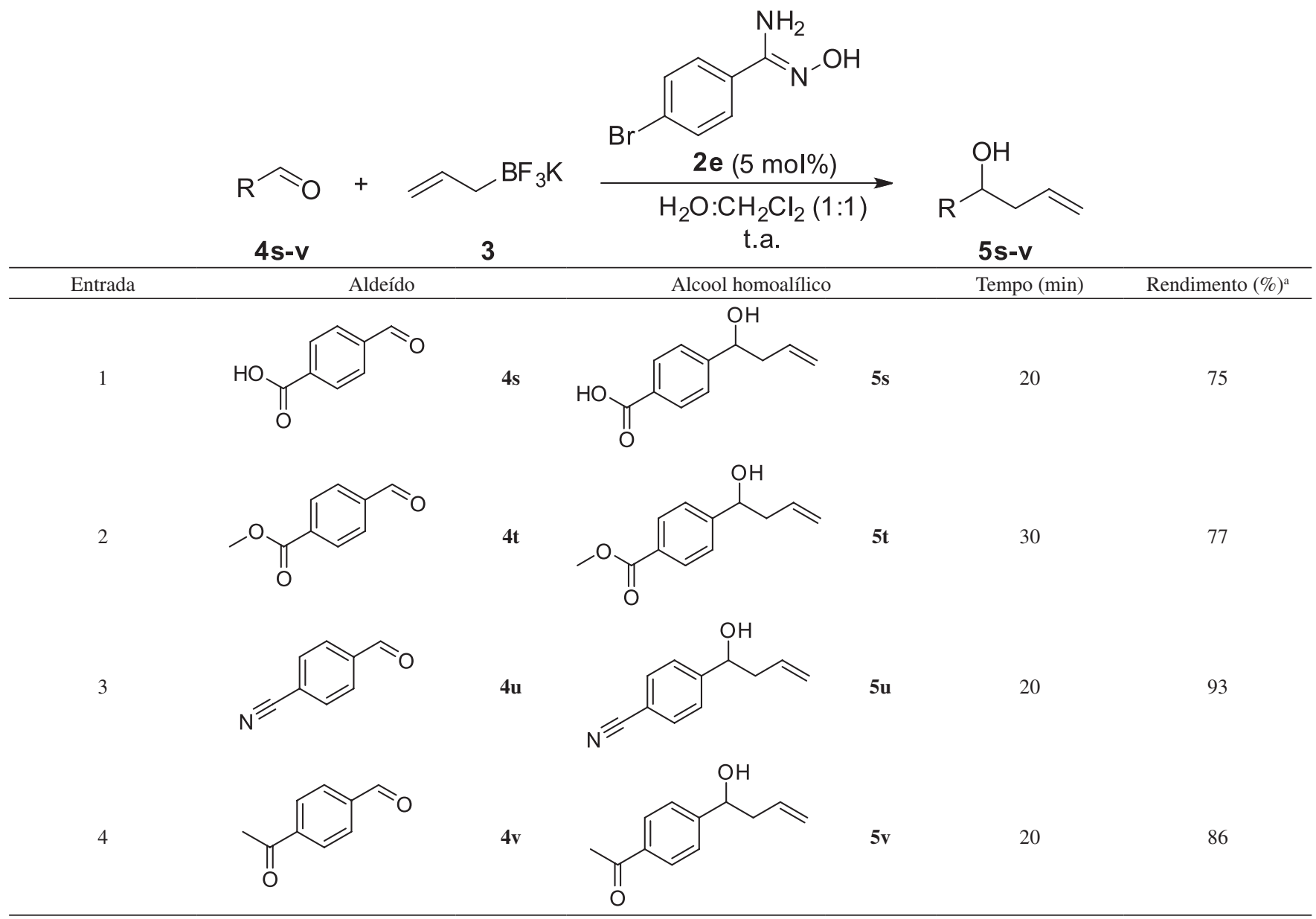

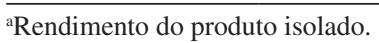

Tabela 7. Avaliação de diferentes promotores na síntese do composto $\mathbf{5 w}$ através da reação de alilação do composto $\mathbf{4 w}$ pelo aliltrifluoroborato de potássio (3)

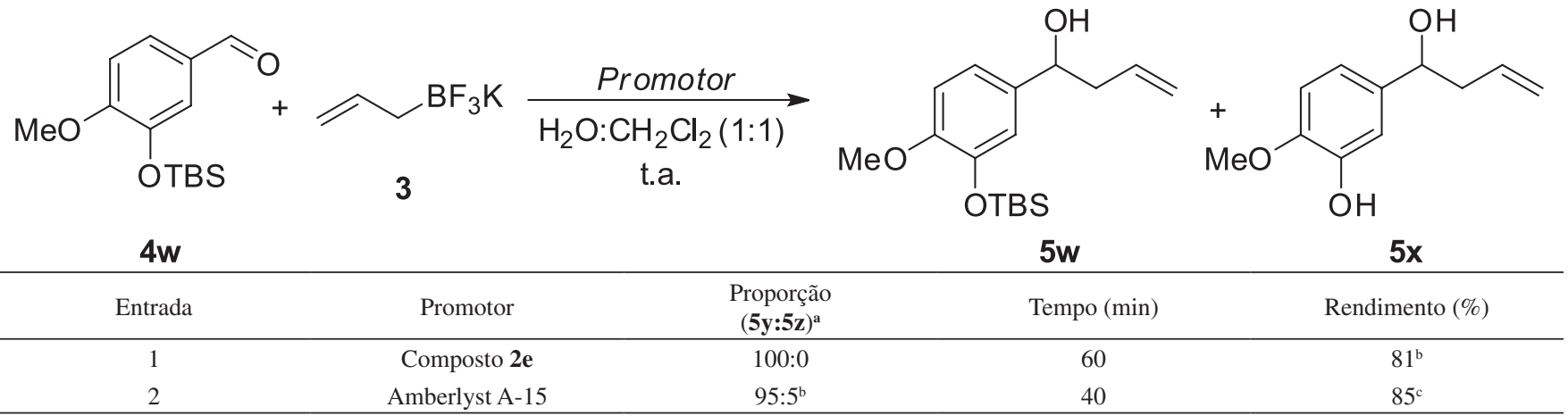

${ }^{\mathrm{a} P r o p o r c ̧ a ̃ o ~ d e t e r m i n a d a ~ p o r ~ c r o m a t o g r a f i a ~ g a s o s a . ~}{ }^{\mathrm{b}}$ Rendimento do produto isolado. ${ }^{\mathrm{c}}$ Rendimento da mistura.

de alilação de aldeídos. A partir do método desenvolvido, os álcoois homoalílicos desejados foram obtidos em rendimentos que variaram de moderados a excelentes, em curtos períodos de tempo, utilizando um sistema bifásico (água:diclorometano) e à temperatura ambiente. Adicionalmente, a reação demonstrou ser quimiosseletiva, podendo ser aplicada como método complementar às demais metodologias já existentes na literatura visando a síntese de moléculas de maior complexidade estrutural.

\section{MATERIAL SUPLEMENTAR}

Os espectros de RMN ${ }^{1} \mathrm{He}{ }^{13} \mathrm{C}$ de todos os compostos sintetizados neste trabalho estão disponíveis em http://quimicanova.sbq.org.br, na forma de arquivo PDF, com acesso livre.

\section{AGRADECIMENTOS}

Os autores agradecem ao CNPq (447361/2014-7), ao PRONEM 
(APQ-0476-1.06/14) pelo suporte financeiro e à CAPES pela bolsa concedida. À Central Analítica do Departamento de Química Fundamental da Universidade Federal de Pernambuco pelas análises dos compostos sintetizados. Ao aluno de mestrado C. R. Dantas pela montagem do resumo gráfico.

\section{REFERÊNCIAS}

1. Neves Filho, R. A. W.; da Silva Alves, D. C. B.; dos Anjos, J. V.; Srivastava, R. M.; Synth. Commun. 2013, 43, 2596; Cai, J.; Wei, H. T.; Hong, K. H.; Wu, X. Q.; Cao, M.; Zong, X.; Li, L. S.; Sun, C. L.; Chen, J. Q.; Ji, M.; Eur. J. Med. Chem. 2015, 96, 1; Barros, C. J. P.; de Souza, Z. C.; de Freitas, J. J. R.; da Silva, P. B. N.; Militão, G. C. G.; da Silva, T. G.; Freitas, J. C. R.; de Freitas Filho, J. R.; J. Chil. Chem. Soc. 2014, 59, 2359; de Freitas, J. J. R.; Freitas, J. C. R.; da Silva, L. P.; de Freitas, J. R.; Kimura, G. Y. V.; Srivastava, R. M.; Tetrahedron Lett. 2007, 48, 6195; Jiang, C.-S.; Fu, Y.; Zhang, L. Gong, J.-X.; Wang, Z.-Z.; Xiao, W.; Zhang, H.-Y.; Guo, Y.-W.; Bioorg. Med. Chem. Lett. 2015, 25, 216.

2. Xu, H.; Ma, S.; Xu, Y.; Bian, L.; Ding, T.; Fang, X.; Zhang, W.; Ren, Y.; J. Org. Chem. 2015, 80, 1789; Khalil, K. D.; Al-Matar, H. M.; Molecules 2012, 17, 12225 .

3. Adib, M., Ansari, S.; Mohammadi, A.; Bijanzadeh, H. R.; Tetrahedron Lett. 2010, 51, 30 .

4. Yavolovskii, A. A.; Grishchuk, L. V.; Rakipov, I. M.; Stepanov, D. E.; Ivanov, Y. E.; Kamalov, G. L.; Chem. Heterocycl. Compd. 2013, 48, 1487; Bassayouni, F. A.; Saleh, T. S.; El-Hefnawi, M. M.; El-Moez, S. I. A.; El-Senousy, W. M.; Abdel-Rehim, M. E.; Arch. Pharm. Res. 2012, 35, 2063; Clement, B.; Drug Metab Rev. 2002, 34, 565.

5. Karamtzioti, P.; Papastergiou, A.; Stefanakis, J. G.; Koumbis, A. E.; Anastasiou, I.; Koffa, M.; Fylaktakidou, K. C.; Med. Chem. Commun. 2015 , 6, 719; Kalvinsh, I.; Abele, R.; Golomba, L.; Rubina, K.; Visnevska, J.; Beresneva, T.; Shestakova, I.; Jaschenko, E.; Bridane, V.; Abele, E.; Heterocycl. Lett. 2011, 1, 47; Oresmaa, L.; Kotikoski, H.; Haukka, M.; Oksala, O.; Pohjala, E.; Vapaatalo, H.; Moilanen, E.; Vainiotalo, P.; Aulaskari, P.; Eur. J. Med. Chem. 2006, 41, 1073; Bouhlel, A.; Curti, C.; Dumètre, A.; Laget, M.; Crozet, M. D.; Azas, N.; Vanelle, P.; Bioorg. Med. Chem. 2010, 18, 7310; Fylaktakidou, K. C.; Hadjipavlou-Litina, D. J.; Litinas, K. E.; Varella, E. A.; Nicolaides, D. N.; Curr. Pharm. Des. 2008, 14, 1001; Doulou, I.; Kontogiorgis, C.; Koumbis, A. E.; Evgenidou, E.; Hadjipavlou-Litina, D.; Fylaktakidou, K. C.; Eur. J. Med. Chem. 2014, 80, 145 .

6. Hu, C. G.; Wang, S. L.; Wu, Z. Z.; Zao, Z.; Li, G.; Revista Materia 2014 19, 350; Mahurin, S. M.; Gorka, J.; Nelson, K. M.; Mayes, R. T.; Dai, S.; Carbon 2014, 67, 457; Shoushtari, A. M.; Zargaran, M.; Abdouss, M.; J. Appl. Polym. Sci. 2006, 101, 2202; Zulfiqar, S.; Karadas, F.; Park, J.; Deniz, E.; Stucky, G. D.; Jung, Y.; Atilhan, M.; Yavuz, C. T.; Energ. Environ. Sci. 2011, 4, 4528; Huang, F.; Xu, Y.; Liao, S.; Yang, D.; YouLo, Q.; Wei, Q.; Materials 2013, 6, 969.

7. Saulter, J. Y.; Kurian, J. R.; Trepanier, L. A.; Tidwell, R. R.; Bridges, A. S.; Boykin, D. W.; Stephens, C. E.; Anbazhagan, M.; Hall, J. E.; Drug Metab. Dispos. 2005, 33, 1886; Cohrs, B.; Zhao, Y.; Lutzen, U.; Culman, J.; Clement, B.; Zuhavra, M.; Int. J. Pharm. 2014, 477, 167.

8. Metwally, S. S.; Ayoub, R. R.; Aly, H. F.; Sep. Sci. Technol. 2013, 48, 1830; Liu, X.; Chen, H.; Wang, C. H.; Qu, R. J.; Ji, C. N.; Sun, C. M.; Xu, Q.; Polymer. Adv. Tech. 2011, 22, 2032; Zhao, Y.; Li, J.; Zhao, L.; Zhang, S.; Huang, Y.; Wu, X.; Wang, X.; Chem. Eng. J. 2014, 235, 275; Coskun, R.; Dilci, Y.; J. Macromol. Sci., Part A: Pure Appl. Chem. 2014, 51,767 .
9. Wu, Z.-C.; Huang, Y.; Lu, Y.-N.; Tao, T.-X.; Zhang, Z.; Catal. Commun. 2012, 29, 158 .

10. Thadani, A. N.; Batey, R. A.; Tetrahedron Lett. 2003, 44, 8051; Barbosa, F. C. G.; Freitas, J. C. R.; Melo, C. F.; Menezes, P. H.; Oliveira, R. A.; Molecules 2012, 17, 14099; Mass, A.; Acocella, M. R.; De Sio, V.; Villano, R.; Scettri, A.; Tetrahedron: Asymm. 2009, 20, 202; Arndt, M.; Reinhold, A.; Hilt, G.; J. Org. Chem. 2010, 75, 5203.

11. Su, B.; Zhang, H.; Deng, M.; Wang, Q.; Org. Biomol. Chem. 2014, 12, 3616; de Souza, R. F. M.; Areias, M. C. C.; Bieber, L. W.; Navarro, M.; Green Chem. 2011, 13, 1118.

12. Chen, M.; Roush, W. R.; J. Org. Chem. 2013, 78, 3; Roush, W.; Palkowitz. A.; J. Am. Chem. Soc. 1987, 109, 953.

13. Sang, F.; Feng, P.; Chen, J.; Ding, Y.; Duan, X.; Zhai, J.; Ma, X.; Zhang, B.; Zhang, Q.; Lin, J.; Chen, Y.; Eur. J. Med. Chem. 2013, 68, 321.

14. Janetzko, J.; Batey, R. A.; J. Org. Chem. 2014, 16, 7415.

15. de Fátima, A.; Kohn, L. K.; de Carvalho, J. E.; Pilli, R. A.; Bioorg. Med. Chem. 2006, 14, 622; Nahra, F.; Riant, O.; J. Chem. Educ. 2015, 92, 179.

16. Zakarian, A.; Batch, A.; Holton, R. A.; J. Am. Chem. Soc. 2013, 125, 7822.

17. Fleury, L. M.; Ashfeld, B. L.; Tetrahedron Lett. 2010, 51, 2427; Yamamoto, Y.; Asao, N.; Chem. Rev. 1993, 93, 2207; Denmark, S. E.; Fu, J.; Chem. Rev. 2003, 103, 2763; Omae, I.; Applications of Organometallic Compounds, John Wiley and Sons: Chichester, 1998; Li, S.; Wang, J.-X.; Wen, X.; Ma, X.; Tetrahedron 2011, 67, 849.

18. Stefani, H. A.; Cella, R.; Vieira, A. S.; Tetrahedron 2007, 63, 3623; Darses, S.; Genet, J.-P.; Chem. Rev. 2008, 108, 288; Alves, D.; Pena, J. M.; Vieira, A. S.; Botteselle, G. V.; Guadagnin, R. C.; Stefani, H. A.; J. Braz. Chem. Soc. 2009, 20, 988; Nascimento, W. S.; Oliveira, J. L.; Freitas, J. C. R.; Navarro, M.; Menezes, P. H.; Synthesis 2014, 2579; Lennox, A. J. J.; Lloyd-Jones, G. C.; Angew. Chem., Int. Ed. 2012, 51, 9385.

19. Nowrouzi, F.; Thadani, A. N.; Batey, R. A.; Org. Lett. 2009, 11, 2631; Ramadhar, T. R.; Bansagi, J.; Batey, R. A.; J. Org. Chem. 2013, 78, 1216; Freitas, J. C. R.; de Oliveira, C. K.; Cunha, E. C.; Malvestiti, I.; Alves, S.; Longo, R. L.; Menezes, P. H.; Tetrahedron Lett. 2013, 54, 1558; Nakamura, H.; Shimizu, K.; Tetrahedron Lett. 2011, 52, 426; Thadani, A. N.; Batey, R. A.; Org. Lett. 2002, 4, 3827; Matsuoka, H.; Kondo, K.; Tetrahedron Lett. 2009, 50, 2320; Lautens, M.; Maddess, M. L.; Org. Lett. 2004, 6, 1883.

20. Perrin, D. D.; Amarego, W. L. F.; Purification of Laboratory Chemicals, Pergamon Press: Oxford, 1996.

21. Still, W. C.; Kahn, M.; Mitra, A.; J. Org. Chem. 1978, 43, 2923.

22. Srivastava, R. M.; Brinn, I. M.; Machuca-Herrera, J. O.; Faria, H. B.; Carpenter, G. B.; Andrade, D.; Venkatesh, C. G.; Morais, L. P. F.; J. Mol. Struct. 1997, 406, 159; da Silva, R. L. Dissertação de Mestrado, Universidade Federal Rural de Pernambuco, Brasil, 2015; Parra, M.; Hidalgo, P.; Alderete, J.; Liq. Cryst. 2005, 32, 449.

23. Barros, C. J. P.; de Freitas, J. J. R.; de Oliveira, R. N.; Freitas Filho, J. R.; J. Chil. Chem. Soc. 2011, 56, 721.

24. Pavia, D. L.; Lampman, G. M.; Kriz, G. S.; Vyvyan, J. R.; Introdução à Espectroscopia, CENGAGE Learning: São Paulo, 2010.

25. Couto, T. R.; Freitas, J. C. R.; Cavalcanti, I. H.; Oliveira, R. A.; Menezes, P. H.; Tetrahedron 2013, 69, 7006. 